\title{
Von der Allgemeinen Erziehungswissenschaft zur Empirischen Bildungsforschung?
}

\section{Eine Analyse von Beiträgen der Zeitschrift für Erziehungswissenschaft}

\section{Andrea Westphal · Olaf Zawacki-Richter}

Eingegangen: 12. Februar 2020 / Überarbeitet: 21. November 2020 / Angenommen: 9. März 2021 /

Online publiziert: 30. März 2021

(C) Der/die Autor(en) 2021

Zusammenfassung Der vorliegende Beitrag beschäftigt sich mit den Publikationen, die in der Zeitschrift für Erziehungswissenschaft (ZfE) in den Jahren 1998-2017 veröffentlicht wurden. Angesichts der Veränderungen in der erziehungswissenschaftlichen Forschungslandschaft in der jüngeren Vergangenheit untersuchen wir, inwiefern sich eine veränderte Schwerpunktsetzung auch in den Beiträgen der ZfE nachweisen lassen. Dazu führen wir zunächst eine quantitative Textanalyse durch und identifizieren die häufigsten sowie die charakteristischen Bigramme (Zweiwortsequenzen) in vier aufeinanderfolgenden Fünfjahres-Abschnitten (1998-2002, 2003-2007, 2008-2012, 2013-2017). Zudem prüfen wir, inwiefern bestimmte Wortstämme (bspw. ,erziehungswissenschaft“, ,bildungsforsch“, ,didakt“) über die Jahre hinweg häufiger auftreten. Schließlich erstellen wir mit dem Textmining Tool Leximancer ${ }^{\mathrm{TM}}$ concept maps, die Hinweise auf die semantische Struktur der Themengebiete und Schlüsselkonzepte geben. Die Ergebnisse deuten darauf hin, dass im gesamten Zeitraum mehrheitlich Beiträge mit empirischem Fokus publiziert wurden, ein inhaltlicher Fokus auf sozialen Aspekten von Bildung lag und die Beschäftigung mit der allgemeinen Erziehungswissenschaft abnahm.

Schlüsselwörter Zeitschriftenanalyse · Wissenschaftsforschung · Inhaltsanalyse · Quantitative Textanalyse $\cdot$ Empirische Wendung

\footnotetext{
Dr. A. Westphal $(\bowtie)$

Empirische Unterrichts- und Interventionsforschung, Universität Potsdam, Karl-Liebknecht-Str. 24-25, 14476 Potsdam, Deutschland

E-Mail: andrea.westphal@uni-potsdam.de

Prof. Dr. O. Zawacki-Richter

Institut für Pädagogik, Center für lebenslanges Lernen (C3L), Carl von Ossietzky Universität Oldenburg, Ammerländer Heerstraße 138, 26129 Oldenburg, Deutschland

E-Mail: olaf.zawacki.richter@uni-oldenburg.de
} 


\title{
From general pedagogy to empirical educational research?
}

An analysis of contributions to the journal Zeitschrift für Erziehungswissenschaft

\begin{abstract}
The present study examines articles published in Zeitschrift für Erziehungswissenschaft (ZfE_-Journal of Educational Sciences) between 1998 and 2017, to discover whether the thematic focus of the articles it published during this period changed over time. To do this, we scrutinized articles in four consecutive fiveyear periods: 1998-2002, 2003-2007, 2008-2012, 2013-2017. Using the titles and abstracts of 821 articles published between 1998 and 2017, we conducted a quantitative text analysis and identified the most frequent and most characteristic bigrams that emerged during these periods. In addition, we looked at whether specific word stems (e.g., "erziehungswissenschaft", "bildungsforsch", "didakt") began to occur more frequently over the two decades under study. Finally, we used the text mining tool Leximancer ${ }^{\mathrm{TM}}$ to construct concept maps that point to the semantic structure of the topics and key concepts in the analyzed articles. The results indicate that, over the course of this twenty-year period, the ZfE predominantly published empirical articles with a focus on the social aspects of education, but that its focus on research into general pedagogy decreased in frequency.
\end{abstract}

Keywords Content analysis - Journal analysis - Quantitative text analysis - Science studies

\section{Einführung}

Die erziehungswissenschaftliche Forschungslandschaft war in den letzten zwanzig Jahren weitreichenden Veränderungen unterworfen, die sich nicht zuletzt in der inhaltlichen und methodischen Ausrichtung der geförderten Forschungsprojekte (Köller 2014) und in der universitären Stellenlandschaft widerspiegeln (Krüger et al. 2008; Schmidt-Hertha und Tippelt 2014). Angesichts dieser Veränderungen stellt sich die Frage, inwiefern sich auch die inhaltliche Schwerpunktsetzung erziehungswissenschaftlicher Fachpublikationen verändert hat. Analysen von Qualifikationsarbeiten in Hochschulschriftenverzeichnissen und von Publikationen der Literaturdatenbank FIS-Bildung deuten aber auf eine weitgehende Stabilität der inhaltlichen Ausrichtung der Forschungsarbeiten hin (Dees und Botte 2013b; Kauder 2014). Im vorliegenden Beitrag untersuchen wir anlässlich des 20-jährigen Jubiläums der Zeitschrift für Erziehungswissenschaft ( $Z f E$ ), inwiefern sich die Verwendung von Begrifflichkeiten in den Fachartikeln - die im Zeitraum von 1998-2017 in der ZfE veröffentlicht wurden - verändert hat und möglicherweise Veränderungstendenzen widerspiegeln können, die in der erziehungswissenschaftlichen Forschungslandschaft $\mathrm{zu}$ beobachten sind.

Befragungen der Deutschen Gesellschaft für Erziehungswissenschaft (Röbken et al. 2013) und erziehungswissenschaftlicher Institutionen (Dees 2013) sowie zitationsbasierte Verfahren zur Messung der Qualität von Zeitschriften - wie die Aufnahme in den „Social Sciences Citation Index“ (SSCI) und das Ranking als hochrangigste deutschsprachige Zeitschrift der Kategorie „Education and Educational 
Research“" vom Web of Science ${ }^{1}$ - deuten darauf hin, dass sich die ZfE seit ihrem erstmaligen Erscheinen vor zwanzig Jahren zu einem der wichtigsten deutschsprachigen Publikationsorgane für die Erziehungswissenschaft entwickelt hat. Das erste Editorial der ZfE im Jahr 1998 trug den Titel „Eine neue erziehungswissenschaftliche Zeitschrift!“ (Lenzen 1998) und verdeutlicht, dass die Herausgeber/innen nicht weniger als eine Erneuerung der erziehungswissenschaftlichen Publikationspraxis anstrebten. Mit der neuen Zeitschrift sollte die Qualität und Internationalität der erziehungswissenschaftlichen Forschung gestärkt werden, die am Ende des 20. Jahrhunderts kaum internationale Debatten in die deutschsprachige Forschung einband oder gar an internationalen Diskursen teilnahm (Gogolin et al. 2013). Obwohl die Erziehungswissenschaft in Deutschland das zweitgrößte Universitätsfach darstellte, war ihr Anteil an der Forschungsförderung bspw. durch die Deutsche Forschungsgemeinschaft gering und das Ansehen erziehungswissenschaftlicher Forschungsleistungen begrenzt (Gogolin et al. 2013). Während die Drittmitteleinnahmen je Professur in den Erziehungswissenschaften im Jahr 2017 bei etwa $97.000 €$ lagen und damit bspw. höher waren als in den Wirtschafts- und Politikwissenschaften, lagen sie im Jahr 1995 bei nur $19.000 €$ und waren deutlich geringer als in diesen beiden Vergleichsdisziplinen (Schmidt-Hertha und Müller 2020)². Mögliche Ursachen wurden in der fehlenden Qualitätskontrolle für erziehungswissenschaftliche Publikationen gesehen - bspw. waren Peer-Review-Prozesse unüblich, obwohl sie sich in der internationalen Forschung bereits etabliert hatten - aber auch in der ,Verstreuung von Forschungsergebnissen in einer unübersehbaren Zahl von Publikationsorganen mit jeweils kleiner Leserschaft" (Gogolin et al. 2013, S. 8). Eine weitere Herausforderung lag in der Vielfalt der erziehungswissenschaftlichen Subdisziplinen, die sich jeweils mit unterschiedlichen Themengebieten befassten (Gogolin et al. 2013). Daraus resultierte das Anliegen ,ein repräsentatives wissenschaftliches Fachorgan für die gesamte Erziehungswissenschaft“ zu schaffen (Lenzen 1998, S. 3), das international anschlussfähig sein, Qualitätssicherung durch einen double-blind PeerReview-Prozess betreiben und aktuelle Forschungsbefunde der Erziehungswissenschaft und ihrer Nachbardisziplinen versammeln sollte (Rost 2013).

Die allgemeine Erziehungswissenschaft „,beansprucht nicht mehr die umfassende Theorie der Erziehung für alle Teilbereiche der Pädagogik vorzustellen“ (Schulze 2002, S. 129). Stattdessen ,versteht [sie] sich selber als Teildisziplin neben vielen anderen, allerdings als Spezialist für das Allgemeine in der Erziehungswissenschaft" und ,untersucht allgemeine den Bereich einer einzelnen Teildisziplin überschreitende Aspekte, Konzepte und Probleme - nicht nur theoretisch reflektierend, auch empirisch forschend“" (Schulze 2002, S. 129). Dabei hat sich die Schwerpunktsetzung der erziehungswissenschaftlichen Forschung in den letzten beiden Jahrzehnten maßgeblich verändert, insbesondere durch die deutliche Stärkung der empirischen

\footnotetext{
1 Siehe aber Botte (2014) für Kritik an diesen traditionellen bibliometrischen Tools und mögliche Alternativen.

2 Bei der Interpretation der absoluten Summe ist zu berücksichtigen, dass die Drittmitteleinnahmen auch in anderen Disziplinen stark angestiegen sind (Schmidt-Hertha und Müller 2020).
} 
Bildungsforschung ${ }^{3}$ (Köller 2014). Wenngleich der vorliegende Beitrag keine vollständige Entwicklung der Erziehungswissenschaft nachzeichnen kann (für einen Überblick siehe Horn 2003; Keiner 1999), sei darauf hingewiesen, dass es bereits in den 1960er- und 1970er-Jahren einige Bemühungen darum gab, ,das textorientierte geisteswissenschaftlich-hermeneutische Selbstverständnis der akademischen Pädagogik zu ergänzen oder nach anderer Sichtweise zu ersetzen durch ein empirischsozialwissenschaftliches Selbstverständnis“ (Fatke und Oelkers 2014, S. 8). Beispielsweise plädierte Roth in seiner Antrittsvorlesung an der Universität Göttingen im Jahr 1962 für eine „,realistische[n] Wendung in der pädagogischen Forschung“, die sich zunehmend auch ,empirische[n] Methoden zur Beschreibung der Erziehungswirklichkeit“" bedienen sollte (Fatke und Oelkers 2014, S. 8-9). Köller (2014) konstatiert allerdings, dass diese realistische Wendung kaum umgesetzt wurde und „zum Beginn der 1990er-Jahre das Hauptinteresse erziehungswissenschaftlicher Forschung auf der Entwicklung und Erprobung von Modellen zur Optimierung der Arbeit in Einzelschulen und dem Entwurf didaktischer Modelle und deren Einführung in die Unterrichtspraxis lag“ (S. 102). Infolge der öffentlichen Aufmerksamkeit auf die Publikation der TIMSS- und PISA-Befunde um die Jahrtausendwende wurde - u. a. durch den Beschluss der Kultusministerkonferenz, dass Deutschland sich zukünftig an internationalen Schulleistungsstudien beteiligen solle - eine zweite empirische Wende bzw. realistische Wendung (Roth 1963) hin zu einer ,evidence-based education" (Gräsel 2011) in der erziehungswissenschaftlichen Forschung eingeleitet (Köller 2014). Diese verstärkte Orientierung an empirischer Forschung spiegelte sich auch in der Vergabe von Fördermitteln (Köller 2014; siehe auch Schmidt-Hertha und Müller 2020) und in der Profilausrichtung von neu geschaffenen Professuren wider (Schmidt-Hertha und Tippelt 2014). So legte die Deutsche Forschungsgemeinschaft (DFG) die beiden Programme „Bildungsqualität von Schule“ (BIQUA, von 1999-2006) und „Kompetenzmodelle zur Erfassung individueller Lernergebnisse und zur Bilanzierung von Bildungsprozessen“ (2007-2015) auf. Auch das Bundesministerium für Bildung und Forschung (BMBF) startete entsprechende Förderinitiativen, wie das „Rahmenprogramm Bildungsforschung“, das nicht nur spezifische Forschungsvorhaben unterstützen, sondern das gesamte Forschungsfeld strukturell fördern sollte. Zudem wurden allein zwischen 2003 und 2006 insgesamt 34 Professuren im Bereich der empirischen Bildungsforschung ausgeschrieben (Krüger et al. 2008). Eine Analyse der Forschungsprojekte, die im Sozialwissenschaftlichen Forschungsinformationssystem (SOFIS) des Leibniz-Instituts für Sozialwissenschaften verzeichnet sind, ergab entsprechend auch, dass der Anteil der verzeichneten Projekte mit bildungswissenschaftlichem Fokus zwischen 1995 und 2006 anstieg (Sondergeld und Botte 2013). Die empirische Wendung ging dabei einher mit einer Öffnung gegenüber anderen Wissenschaftsdisziplinen. Die von den empirischen Forscherinnen und Forschern verwendeten Methoden, aber auch die Theorien, die

\footnotetext{
${ }^{3}$ Eine Definition der empirischen Bildungsforschung formuliert Gräsel (2011). Sie weist darauf hin, dass die empirische Bildungsforschung ,im Kern [fragt], wie Bildungsprozesse verlaufen, wer welche Qualifikationen und Kompetenzen im Bildungssystem erwirbt, wovon dieser Qualifikations- und Kompetenzerwerb abhängig ist, und welche Auswirkungen er hat“ (S. 13). Gräsel (2011) bezeichnet die Problemorientierung, die Interdisziplinarität und die Verwendung empirischer Forschungsmethoden als die Kernmerkmale der empirischen Bildungsforschung.
} 
sie ihren Untersuchungen zugrunde legen, stammen oftmals aus Soziologie, Psychologie und Ökonomie und sind nicht mehr nur genuin erziehungswissenschaftlich (Gräsel 2011; Fatke und Oelkers 2014). Gräsel (2011) stellte fest, dass sich die beschriebenen Veränderungen auch in der Publikationslandschaft widerspiegeln. So seien vermehrt Publikationen und Publikationsorgane zu verzeichnen, die sich in der empirischen Bildungswissenschaft verorten. Im Jahr 2012 wurde zudem die Gesellschaft für Empirische Bildungsforschung (GEBF) gegründet. Im Folgenden werden einige Untersuchungen skizziert, die prüfen, inwiefern sich Veränderungen sowohl in den öffentlichen Medien und ihrem Blick auf die Erziehungswissenschaften als auch in der erziehungswissenschaftlichen Publikationslandschaft in den letzten Dekaden tatsächlich empirisch belegen lassen.

\subsection{Inwiefern lassen sich die Veränderungen der erziehungswissenschaftlichen Forschungslandschaft auch in bildungsrelevanten Publikationen nachweisen?}

Anhand einer Analyse von 180.000 bildungsrelevanten Artikeln, die zwischen 1984 und 2015 in öffentlichen Zeitungen und Newslettern, bspw. in der Berliner Zeitung, dem Spiegel oder der ZEIT veröffentlicht wurden, konnten Botte und Rittberger (2016) zeigen, dass in den letzten dreißig Jahren in den öffentlichen Printmedien eine gewisse Themenverschiebung zu verzeichnen war. Die Autoren nutzten die inhaltlichen Schlagwörter, die in der Datenbank ,Zeitungsdokumentation Bildungswesen“" verfügbar waren und ordneten die Aritkel größeren Themenbereichen zu. Beispielsweise wurden die Wortstämme Schul*, Schüler*, Lehrer* und Unterricht dem Themenbereich Schule zugewiesen. Dabei prüften die Autoren, wie sich verschiedene Themenbereiche über die Zeit entwickelten. Sie stellten fest, dass der Bereich Schule anteilig leicht zunahm (31\% der Beiträge zwischen 1991-1998, verglichen mit je 38\% in 1999-2002 und in 2007-2014). Auch der Anteil der Beiträge, die sich mit dem Bereich Vorschule beschäftigten, wuchs marginal an $(1,5 \%$ in 1991-1998; 2,5\% in 1999-2002; $5 \%$ in 2007-2014). Der Bereich Hochschule verzeichnete hingegen eine sinkende Tendenz mit 32\% in 1991-1998, verglichen mit $28 \%$ in 1999-2002 und 26\% in 2007-2014. Diese Ergebnisse geben Aufschluss über die Relevanz von Bildungsthemen in Zeitungen und Zeitschriften, die von einer breiten Öffentlichkeit rezipiert werden. Allerdings können die thematischen Veränderungen nur schwer mit den Veränderungen in der erziehungswissenschaftlichen Forschungslandschaft verknüpft werden.

Inwiefern sich auch die erziehungswissenschaftliche Publikationslandschaft in ihrer inhaltlichen und methodischen Ausrichtung in den letzten zwanzig Jahren verändert hat, wurde in einigen wenigen empirischen Aufsätzen beleuchtet. Im BMBFProjekt „Bildungsforschung in Deutschland - eine Situationsanalyse“ wurde eine umfangreiche Auswertung der Publikationen vorgenommen, die in der Literaturdatenbank FIS-Bildung gelistet sind (Weishaupt und Rittberger 2013). Die Autoren ermittelten die Veränderung der inhaltlichen Schwerpunkte der gelisteten Publikationen, indem sie die verfügbaren Schlagwörter verschiedenen Forschungsgebieten innerhalb der Erziehungswissenschaften zuordneten, bspw. der Fachdidaktik, der Erwachsenenbildung, der Sonderpädagogik, oder der Bildungsforschung und -theorie. 
Dabei zeigte sich, dass der Anteil der Publikationen, die sich jedem dieser Forschungsgebiete zuordnen ließen im Zeitraum 2003-2007 sehr ähnlich war wie im Zeitraum 1998-2002 (prozentuale Differenzen $\leq 1 \%$; Dees und Botte 2013b). Beispielsweise wurden in beiden Zeiträumen jeweils $10 \%$ aller Publikationen in FISBildung der Kategorie Bildungsforschung und -theorie zugeordnet. Lediglich auf struktureller Ebene fanden sich Unterschiede zwischen diesen Zeiträumen. So stieg der Anteil der elektronischen Publikationen von 4\% im Zeitraum 1998-2002 auf $9 \%$ im Zeitraum 2003-2007, während der Anteil englischsprachiger Publikationen stabil niedrig blieb (2,3\% in 1998-2002 vs. 3,6\% in 2003-2007; Dees und Botte 2013b). Der Anteil der Zeitschriftenaufsätze nahm laut Dees und Botte (2013b) leicht ab (59\% in 1998-2002 vs. 53\% in 2003-2007). Allerdings stellte Botte (2015) - der Publikationen aus mehr als 9000 erziehungswissenschaftlichen, soziologischen und psychologischen Forschungsprojekten auswertete - fest, dass in der Erziehungswissenschaft der Anteil der Zeitschriftenpublikationen gemessen an allen Publikationsformen in den letzten Jahren stärker angestiegen ist (23\% in 1995-1997 vs. 35\% in 2006-2008) als in der Psychologie (34\% in 1995-1997 vs. 39\% in 2006-2008) und der Soziologie (33\% in 1995-1997 vs. $22 \%$ in 2006-2008).

Darüber hinaus untersuchte Kauder (2014), wie sich die inhaltliche und methodische Ausrichtung von Dissertationen zwischen 1945 und 2009 veränderte. Dafür nutzte er die Angaben zu erziehungswissenschaftlichen Dissertationen, die von den Hochschulen in Deutschland, Österreich und der Schweiz an die ZfPäd gemeldet wurden. Seine Auswertungen zeigen, dass empirische Methoden bei Dissertationen, die zwischen 1990 und 2009 entstanden, deutlich häufiger angewandt wurden (insgesamt $40 \%$ aller Dissertationen) als im Zeitraum 1945-1990 (25\%). Der Anteil praxisbezogener Arbeiten stieg ebenfalls leicht an (29\% in 1945-1990 vs. $33 \%$ in 1990-2009). Hingegen sank der Anteil der Dissertationen mit historischer (24\% in 1945-1990 vs. 9\% in 1990-2009) oder theoretischer Ausrichtung (23\% in 1945-1990 vs. $18 \%$ in 1990-2009). Zudem prüfte Kauder (2014), inwiefern die Titel der Dissertationen bestimmte inhaltliche Begriffe wie „unterricht“, „lehrer“, „schüler“, „lernen“, „medien“ oder „migration“ enthielten. Seine Befunde legen nahe, dass die inhaltliche Ausrichtung der Arbeiten im Zeitraum 1990-1999 ähnlich war, wie im Zeitraum 2000-2009 (identische Rangfolge, prozentuale Differenzen $\leq 3 \%$ ).

Diese Arbeiten sind aufschlussreich und werfen zugleich neue Fragen auf. Denn angesichts der maßgeblichen Entwicklungen in der erziehungswissenschaftlichen Forschungslandschaft wäre anzunehmen, dass sich auch die inhaltliche Ausrichtung der erziehungswissenschaftlichen Publikationen deutlich verändert hat. Die Arbeiten von Dees und Botte (2013b) sowie von Kauder (2014) finden jedoch kaum Hinweise auf eine veränderte inhaltliche Schwerpunktsetzung in den fachwissenschaftlichen Publikationen und Qualifikationsarbeiten. Denkbar wäre, dass entsprechende Veränderungen nur dann aufgedeckt werden können, wenn ein Zeitraum von mehr als 10 Jahren betrachtet und zusätzlich zu den recht kurzen und möglicherweise nicht ausreichend informativen Titeln der Arbeit auch die Zusammenfassungen in die Analyse einbezogen werden. Zudem ergibt sich für eine systematische Analyse der deutschsprachigen erziehungswissenschaftlichen Publikationslandschaft die Herausforderung, dass die zur Verfügung stehende Datenbasis einige Defizite auf- 
weist (Abs und Kuper 2020). Insbesondere der Anteil der publizierten Literatur, die in der Datenbank FIS Bildung erfasst ist, wird nach Einschätzung von Abs und Kuper (2020) von Jahr zu Jahr geringer. Die vorliegende Arbeit erhebt nicht den Anspruch auf eine vollständige Analyse der gesamten Publikationsleistung der deutschsprachigen Erziehungswissenschaft. Stattdessen untersuchen wir, inwiefern sich Veränderungen in der inhaltlichen Schwerpunktsetzung der erziehungswissenschaftlichen Publikationen im deutschsprachigen Raum in der ZfE widerspiegeln, die aus Sicht von wissenschaftlich tätigen Erziehungswissenschaftler/innen eines der wichtigsten deutschsprachigen Publikationsorgane der Erziehungswissenschaft darstellt (Dees 2013; Röbken et al. 2013). Das Ziel des vorliegenden Beitrags liegt daher darin, Veränderungen in der Verwendung von Begrifflichkeiten in den publizierten Originalbeiträgen der ZfE - in den Jahren 1998-2017 - aufzuzeigen und ggf. Hinweise auf thematische Trends zu identifizieren ${ }^{4}$.

\section{Methode}

\subsection{Stichprobe}

Die vorliegende Untersuchung basierte auf $N=821$ Originalbeiträgen, die in der ZfE über 20 Jahre (1998-2017) veröffentlicht wurden. Mittels eines Web Scrapings der ZfE-Webseite extrahierten wir automatisiert 862 Beiträge, die in den Jahren 2005-2017 veröffentlicht wurden. Davon wurden 606 Originalbeiträge für die vorliegenden Analysen berücksichtigt, während Editorials, Rezensionen oder Kommentare ausgeschlossen wurden. Darüber hinaus wurden 215 Originalbeiträge aus den Jahren 1998-2004 manuell von der Webseite und den Druckausgaben der ZfE extrahiert, wobei Editorials, Rezensionen oder Kommentare ebenfalls nicht berücksichtigt wurden; ein Beitrag wurde ausgeschlossen, da Abstract und Titel ausschließlich in englischer Sprache verfügbar waren. Im Fokus unserer Untersuchung standen die Abstracts dieser Originalbeiträge. Für $2 \%$ der 821 Originalbeiträge $(n=17)$ lag kein Abstract vor, so dass stattdessen der deutschsprachige Titel in den Analysen berücksichtigt wurde. Von diesen Beiträgen sind 153 (19\%) zwischen 1998 und 2002; $150(18 \%)$ zwischen 2003 und 2007; 212 (26\%) zwischen 2008 und 2012 und 306 (37\%) zwischen 2013 und 2017 entstanden.

\subsection{Methodisches Vorgehen}

Um wesentliche Inhalte und deren thematischen Verschiebungen der $Z f E$ aufzuzeigen, verwendeten wir für den vorliegenden Beitrag drei verschiedene Analyseansätze. Im Vorfeld der Analysen unterteilten wir die Daten in vier Zeitabschnitte (1998-2002, 2003-2007, 2008-2012 und 2013-2017), um die Veränderung von Inhalten über die Zeit zu prüfen. Im ersten Analyseschritt führten wir quantitative Textanalysen u.a. mit dem Package tidytext (Silge und Robinson 2016) in R

\footnotetext{
${ }^{4}$ Beiträge der ZfE aus den Jahren 2001-2010 wurden bereits von Gallmann (2018) mit einer anderen Schwerpunktsetzung - der Identifikation von Unschärfe - analysiert.
} 
durch $^{5}$. Dabei zerlegten wir die Abstracts in Sequenzen von jeweils zwei aufeinander folgenden Wörtern, sogenannten Bigrammen, die einheitlich klein geschrieben waren. Aus diesen Bigrammen schlossen wir diejenigen aus, die im Deutschen häufig vorkommende Wörter (stopwords) enthielten. Dafür verwendeten wir das ,Snowball"“-Lexikon, das im tidytext-Package implementiert ist. Anschließend ermittelten wir, welche Bigramme, in den vier Zeitabschnitten am häufigsten auftraten. Häufig vorkommende Bigramme mit geringer semantischer Information wurden ausgeschlossen (bspw. ,et al.“, ,immer schon“). Für die 20 häufigsten Bigramme pro Zeitabschnitt vereinheitlichten wir die Endungen von Adjektiven und Substantiven (bspw. ersetzten wir ,allgemeiner Erziehungswissenschaft“ und ,allgemeinen Erziehungswissenschaft" durch ,allgemeine Erziehungswissenschaft"). Anschließend bestimmten wir die term frequency-inverse document frequency (tf-idf) für die $\mathrm{Bi}$ gramme in jedem der vier Zeitabschnitte. Das $t f$-idf-Maß ist das Produkt aus der term frequency (relative Häufigkeit des Bigramms) und der inverse document frequency, die sich beschreiben lässt als $i d f($ term $)=\ln \left(\mathrm{n}_{\text {documents }} / \mathrm{n}_{\text {documents containing term }}\right)$. Hiermit wird also eine Gewichtung vorgenommen, wobei davon ausgegangen wird, dass in einem Dokument weniger häufig vorkommende Wörter eine größere Bedeutung haben als häufig vorkommende Wörter wie z.B. die stopwords (vgl. Silge und Robinson 2016).

Vereinfacht lagen uns somit vier „Dokumente“ - eines für jeden Zeitabschnitt vor, so dass das $t f-i d f$-Maß die Relevanz eines Bigramms innerhalb eines Zeitabschnitts im Vergleich zum gesamten Veröffentlichungszeitraum indiziert. Bigramme mit hohen $t f$-idf-Werten sind demnach besonders charakteristisch für den jeweiligen Zeitabschnitt. Dabei wurden aus den Bigrammen mit den höchsten tf-idfWerten (Ränge eins bis zehn) Bigramme mit geringer semantischer Information ausgeschlossen (bspw. „bislang allerdings“, ,deutlich stärker“) und die Endungen von Adjektiven und Substantiven vereinheitlicht. Beispielsweise ersetzten wir ,qualitativen Methoden“ durch „qualitative Methoden“, um nicht fälschlicherweise zu schließen, dass die grammatikalisch seltenere Form besonders charakteristisch sei.

Im zweiten Analyseschritt verwendeten wir das Package stringr, um für jeden Beitrag zu kodieren, ob bestimmte Wortstämme (bspw. ,erziehungswissenschaft“, „,bildungsforsch“, ,didakt“, „,histor“) im Titel oder Abstract auftraten. Anschließend berechneten wir den mittleren Anteil der Beiträge pro Jahr, die den entsprechenden Wortstamm enthielten. Um kurzzeitige Schwankungen zu glätten und längerfristige Entwicklungen aufzuzeigen, sind in unseren Abbildungen die gleitenden Mittelwerte jeweils über ein Fenster von fünf Jahren dargestellt.

In einem dritten Analyseschritt nutzten wir das Textmining Tool Leximancer ${ }^{\mathrm{TM}}$, um concept maps zu erstellen, die die semantische Struktur der Themengebiete und Schlüsselkonzepte widerspiegeln. Leximancer ${ }^{\mathrm{TM}}$ wurde bereits von verschiedenen Wissenschaftler/innen angewandt, um unterschiedliche Zeitschriften wie das Journal of Cross-Cultural Psychology (Cretchley et al. 2010), die Zeitschrift Distance Education (Zawacki-Richter und Naidu 2016), das Journal of Studies in Interna-

\footnotetext{
5 Ähnliche Analysen sind auch mit dem Inhalts- und Textanalyseprogramm Textpack möglich (Züll und Mohler 1992). Einen Literaturbericht zu Publikationen, die Textpack oder ähnliche Programme der computerunterstützten Inhaltsanalyse nutzen, haben Züll und Landmann (2002) verfasst.
} 


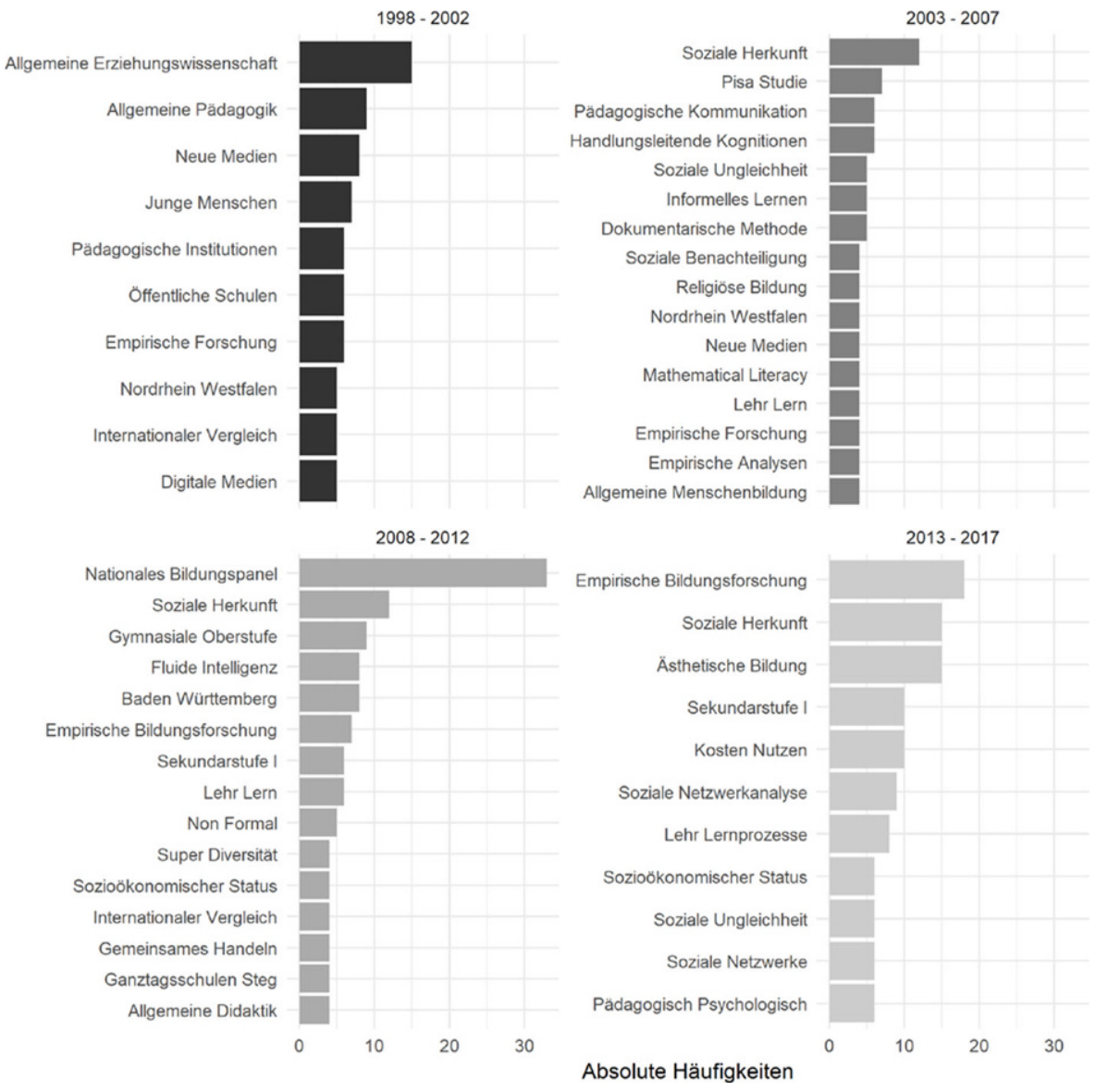

Abb. 1 Die häufigsten Bigramme in den Abstracts der ZfE in den Jahren 1998-2017

tional Education (Bedenlier et al. 2018) oder das Journal of Communication (Lin und Lee 2012) inhaltsanalytisch auszuwerten. Die Software identifiziert auf Basis der Textdaten die am häufigsten vorkommenden Wörter (concepts) und deren Beziehungen untereinander, indem das gemeinsame Auftreten von Wörtern in Texten analysiert wird (relational analyis). Konzepte, die häufig gemeinsam auftreten, werden in einem Cluster oder einer thematischen Region zusammengefasst, die den Namen des am häufigsten auftretenden Konzepts erhält (vgl. Smith und Humphreys 2006). Die Analysen mit der Software Leximancer ${ }^{\mathrm{TM}}$ wurden einmal für den gesamten Zeitraum und separat für die vier Zeitabschnitte durchgeführt.

\section{Ergebnisse}

Im Folgenden beschreiben wir zunächst, wie sich die Anzahl der Autor/innen pro ZfE-Beitrag und die Beteiligung von Autorinnen (im Vergleich zu Autoren) entwi- 


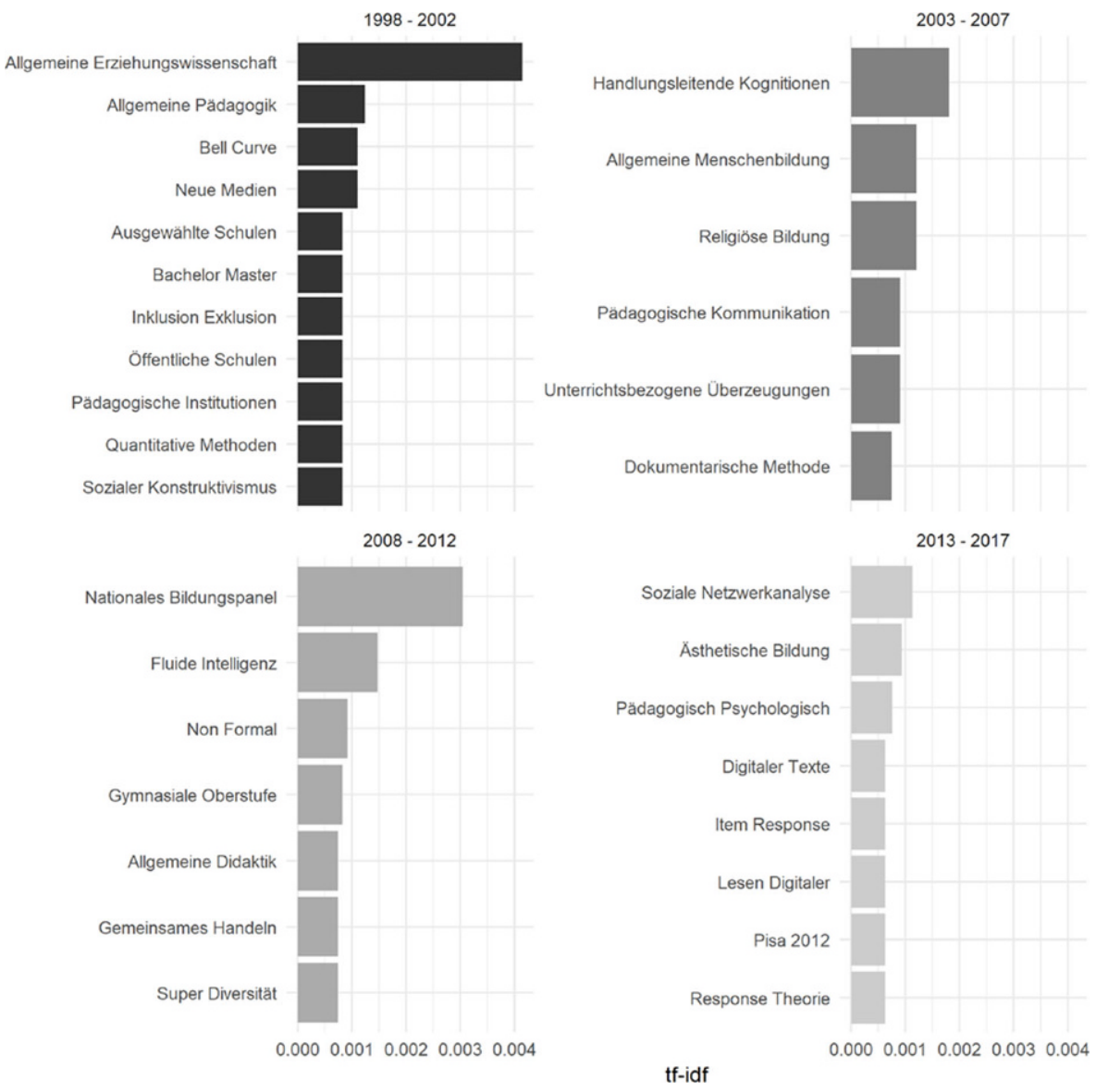

Abb. 2 Bigramme mit den höchsten tf-idf-Werten in den Abstracts der ZfE in den Jahren 1998-2017

ckelt hat und welche Wissenschaftler/innen und Institutionen besonders häufig an Beiträgen der ZfE mitgewirkt haben ${ }^{6}$. Im nächsten Schritt stellen wir die häufigsten Bigramme für die Fünfjahresabschnitte dar sowie die charakteristischsten Bigramme, die in einem Zeitraum sehr häufig vorkamen, in den anderen Zeiträumen jedoch selten waren. Daran anschließend zeigen wir, wie sich die Verwendung bestimmter Wortstämme, wie „erziehungswissenschaft“, „,bildungsforsch“, „didakt“, „histor“, „,philosoph“, „ökonom“, „psycholog“, „soziolog“ über die Zeit entwickelt hat. Im letzten Schritt beschreiben wir die semantische Struktur der Themengebiete und Schlüsselkonzepte in ZfE-Beiträgen und ihre Entwicklung über die Zeit.

\footnotetext{
6 Da diese Ergebnisse nicht zentral für die Frage sind, inwiefern sich Entwicklungstendenzen in der inhaltlichen Schwerpunktsetzung erziehungswissenschaftlicher Publikationen in der ZfE widerspiegeln, präsentieren wir diese Tabellen und Abbildungen im Anhang des Beitrags.
} 
30

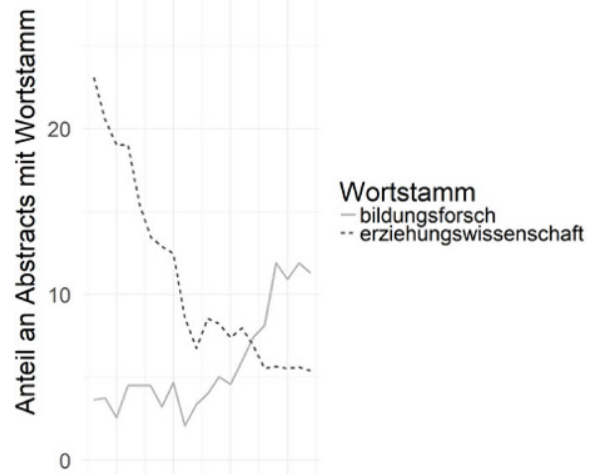

2000200520102015

30

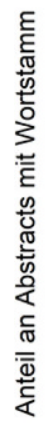

$2000 \quad 2005 \quad 2010 \quad 2015$
30

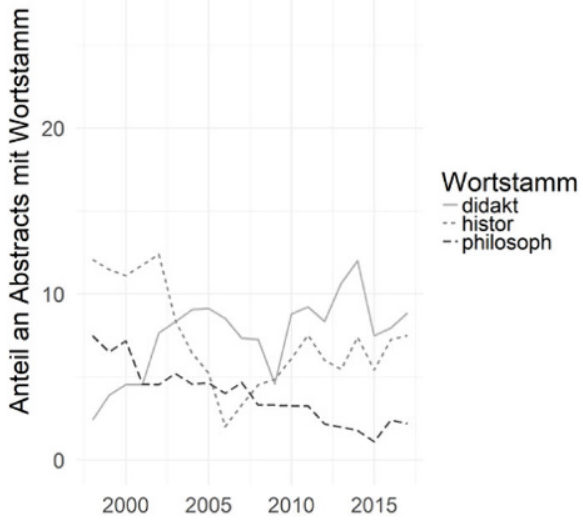

30

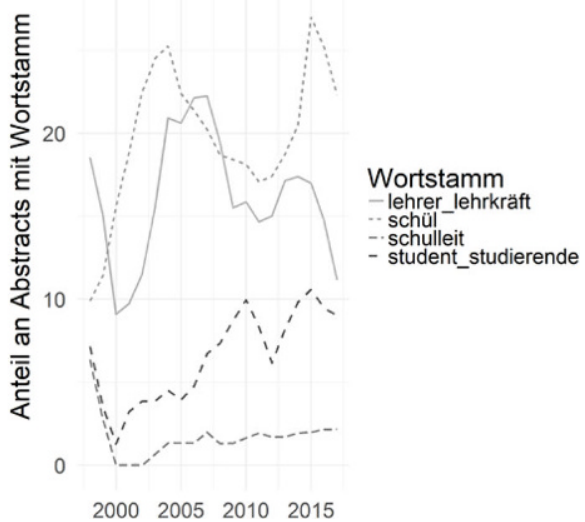

Abb. 3 Anteil an Abstracts der ZfE in den Jahren 1998-2017 mit den Wortstämmen (,,bildungsforsch“, „erziehungswissenschaft“, „didakt“, „histor“, ,philosoph“, „ökonom“, ,psycholog“, ,,soziolog“, „lehrer“ oder ,lehrkraft“, „,schül“, ,,schulleit“, ,,student” oder ,studierende“) (dargestellt sind gleitende Mittelwerte über ein Zeitfenster von fünf Jahren)

\subsection{Deskriptive Ergebnisse zu den Autor/innen der $Z f E$-Beiträgen in den Jahren 1998-2017}

In Abb. 9 und 10 im Anhang ist dargestellt, wie sich die Anzahl der Autorinnen und Autoren entwickelte, die im Zeitraum von 1998-2017 an ZfE-Artikeln beteiligt waren. Es zeigte sich, dass die mittlere Autorenzahl über die Zeit hinweg ansteigt und die Anzahl an Publikationen, die von nur einem Autor oder einer Autorin gestaltet werden, rückläufig ist. Abb. 11 im Anhang verdeutlicht, dass der Anteil an Autorinnen sowie der Anteil an Erstautorinnen über die Zeit zugenommen hat. In Tab. 1 im Anhang ist die institutionelle Zugehörigkeit nach Ländern der an ZfE-Beiträgen beteiligten Autorinnen und Autoren dargestellt. Es wird deutlich, dass am überwiegenden Anteil der Publikationen Autorinnen und Autoren aus Deutschland beteiligt 
Abb. 4 Concept Map erstellt mit dem Textmining Tool Leximancer $^{\mathrm{TM}}$ auf Grundlage der Abstracts der ZfE in den Jahren 1998-2017 $(N=821)$

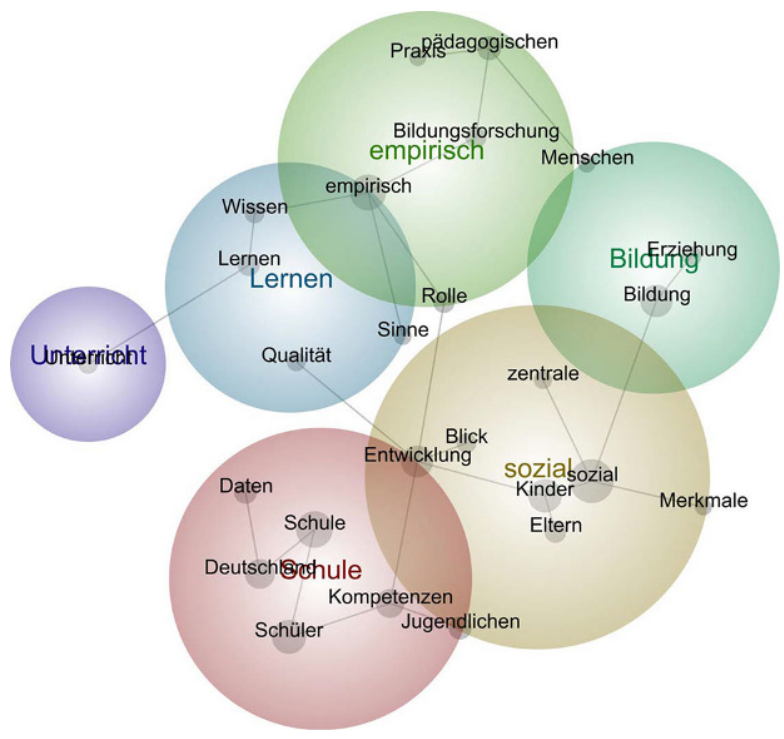

waren. Tab. 2 im Anhang zeigt die institutionellen Zugehörigkeiten nach Einrichtungen der an den $Z f E$-Beiträgen beteiligten Autorinnen und Autoren. Dabei führt die Freie Universität Berlin, gefolgt von der Humboldt-Universität Berlin und der Universität Hamburg. Schließlich findet sich in Tab. 3 im Anhang eine Aufschlüsselung der Autorinnen und Autoren mit der höchsten Anzahl an ZfE-Beiträgen.

Abb. 5 Concept Map erstellt mit dem Textmining Tool Leximancer ${ }^{\mathrm{TM}}$ auf Grundlage der Abstracts der ZfE in den Jahren $1998-2002(N=153)$

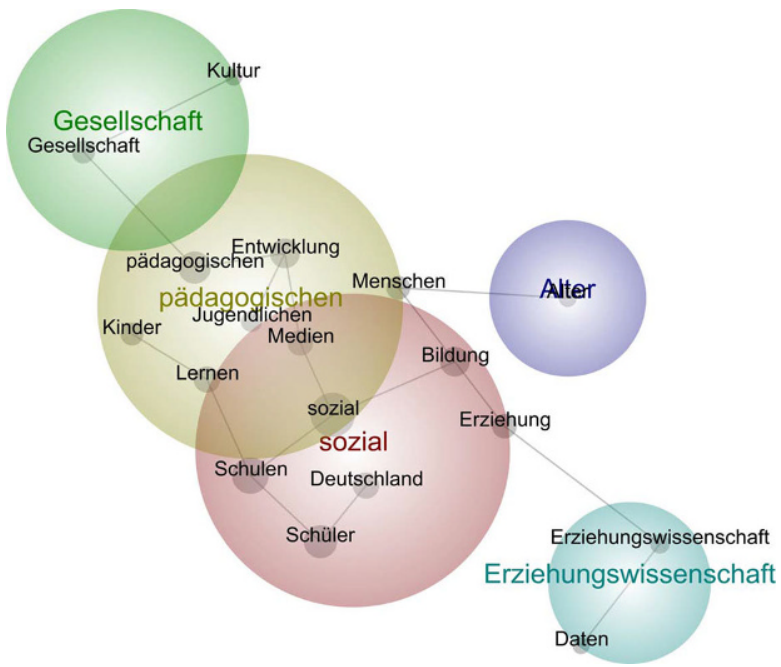




\subsection{Häufige bzw. charakteristische Bigramme in ZfE-Beiträgen in den Jahren 1998-2017}

In Abb. 1 sind die häufigsten Bigramme in den Abstracts der ZfE in den Jahren 1998-2017 dargestellt. Das mit Abstand am häufigsten genannte Bigramm war „Nationales Bildungspanel“, das in den Jahren 2008-2012 insgesamt 33 mal aufgeführt wurde. An zweiter und dritter Stelle folgten die Bigramme „Empirische Bildungsforschung“ ( $n=18$ in 2013-2017), ,Allgemeine Erziehungswissenschaft" ( $n=15$ in 1998-2002), „Ästhetische Bildung“ ( $n=13$ in 2013-2017) und „Soziale Herkunft“ ( $n=13$ in 2013-2017). Auch in den Zeitabschnitten 2003-2007 bzw. 2008-2012 war das Bigramm „Soziale Herkunft“ mit jeweils zwölf Nennungen sehr häufig vertreten.

Darüber hinaus sind in Abb. 2 die Bigramme mit den höchsten tf-idf-Werten dargestellt. Die tf-idf-Werte drücken eine Gewichtung der Bigramme aus. Besonders hoch gewichtet werden diejenigen Bigramme, die in einem Fünfjahresabschnitt häufig vorkommen, während sie in den anderen Fünfjahresabschnitt sehr selten sind. In anderen Worten sind Bigramme mit einem hohen tf-idf-Wert besonders charakteristisch für einen Zeitabschnitt. Das Bigramm mit dem höchsten tf-idf-Wert war „Allgemeine Erziehungswissenschaft“ (tf-idf=0,004 in 1998-2002), gefolgt von „Nationales Bildungspanel“ (tf-idf=0,003 in 2008-2013) und „Handlungsleitende Kognitionen“" (tf-idf=0,002 in 2003-2007). Diese Bigramme waren demnach besonders charakteristisch für den jeweiligen Zeitabschnitt, denn sie traten im jewei-

Abb. 6 Concept Map erstellt mit dem Textmining Tool Leximancer $^{\mathrm{TM}}$ auf Grundlage der Abstracts der ZfE in den Jahren 2003-2007 $(N=150)$

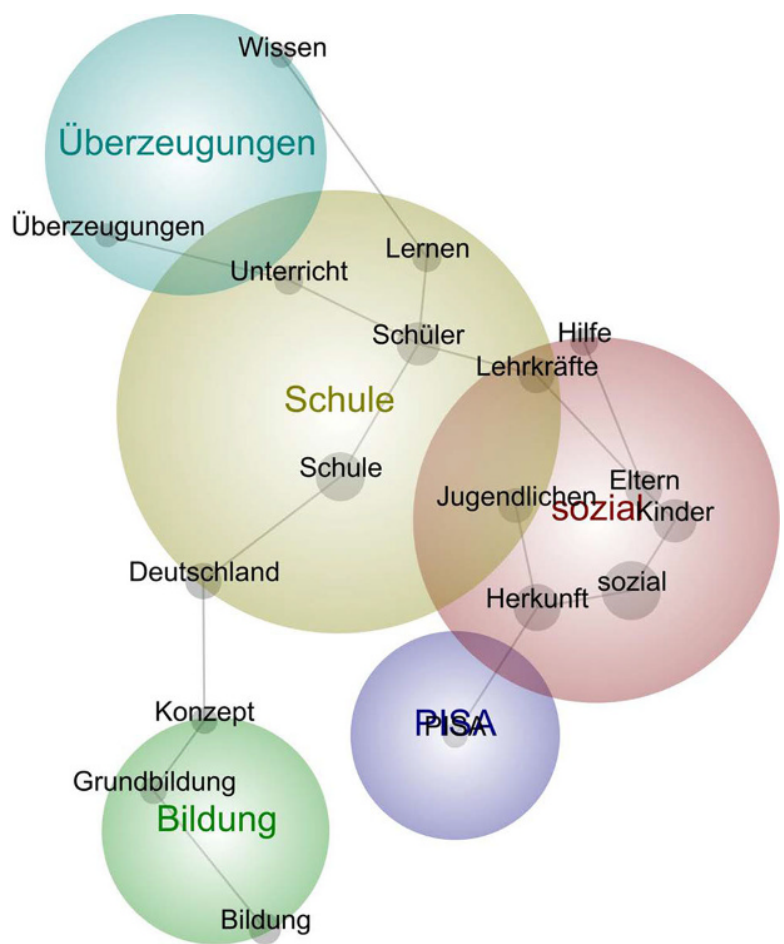


ligen Zeitabschnitt häufig auf, aber in den anderen Zeitabschnitten selten oder gar nicht. Die Abbildung verdeutlicht, dass sich für den Zeitabschnitt 1998-2002 sowie für 2008-2012 jeweils ein besonders charakteristisches Bigramm identifizieren lässt, das einen höheren tf-idf-Wert aufweist als die Bigramme mit zweit- bzw. dritthöchsten tf-idf-Werten. Hingegen weisen die tf-idf-Werte der charakteristischsten Bigramme in den Zeitabschnitten 2003-2007 und 2013-2017 eine geringere Streuung auf, da der jeweils höchste tf-idf-Wert sich weniger stark vom tf-idf-Werten auf Rang 2 unterscheidet (siehe Abb. 2).

\subsection{Spezifische Wortstämme in ZfE-Beiträgen in den Jahren 1998-2017}

In Abb. 3 ist der prozentuale Anteil der Abstracts pro Jahr dargestellt, die spezifische Wortstämme verwendeten. Die Abbildung stellt gleitende Mittelwerte jeweils über ein Zeitfenster von fünf Jahren dar. Die Abbildung zeigt zunächst, dass der Wortstamm ,erziehungwissenschaft“ in den Anfangsjahren der ZfE häufiger genutzt wurde als in späteren Jahren der Zeitschrift. Der Wortstamm ,bildungsforsch“ wurde hingegen in zunehmendem Maße genannt. Darüber hinaus fanden wir für die Wortstämme ,histor“ und ,philosoph“ eine leicht sinkende Tendenz, während für den Wortstamm ,didakt“ eine steigende Tendenz zu verzeichnen war. Auch für den Wortstamm ,soziolog“ ließ sich eine steigende Tendenz identifizieren, während der Wortstamm ,psycholog“ nur marginal häufiger genutzt wurde und der Wortstamm „ökonom“ eine nahezu gleichbleibende Verlaufskurve aufwies.

Schließlich fanden wir, dass die Abstracts der ZfE die Wortstämme „lehrer“ bzw. „lehrkräft“ und „,schül“ sehr viel häufiger verwendeten als die Wortstämme ,student“ bzw. „studierende“ und ,schulleit“. Der Wortstamm ,schulleit“ wurde im Jahr 2005 erstmals von einem Abstract genannt und verzeichnet seither eine zunehmende Tendenz. Die Wortstämme „student“ bzw. ,studierende“ wurden erstmals 2002 angeführt, aber auch in den letzten Jahren nur sehr selten genannt.

Abb. 7 Concept Map erstellt mit dem Textmining Tool Leximancer $^{\mathrm{TM}}$ auf Grundlage der Abstracts der ZfE in den Jahren 2008-2012 $(N=212)$

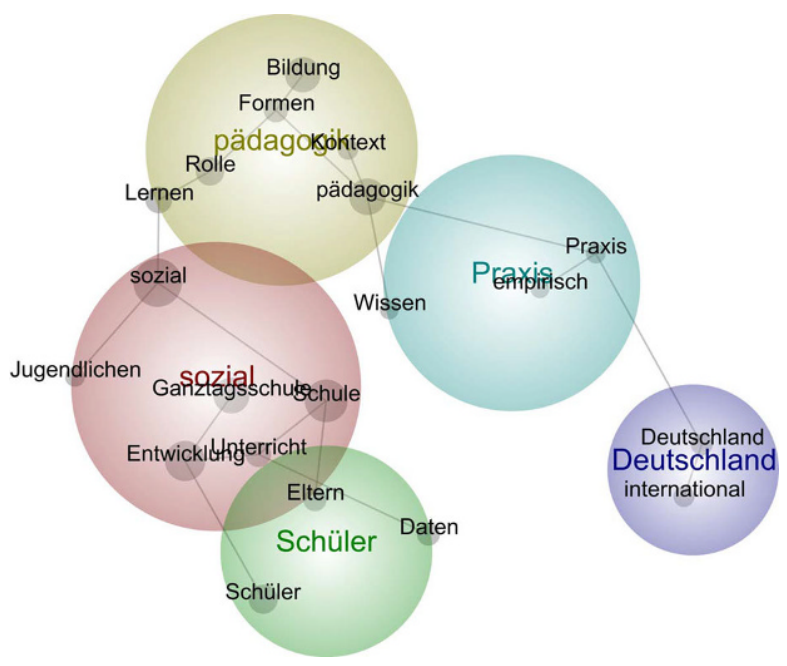




\subsection{Semantische Struktur der Themengebiete und Schlüsselkonzepte in ZfE - Beiträgen und ihre Entwicklung über die Zeit}

Für eine vertiefende Inhaltsanalyse nutzten wir die Software Leximancer ${ }^{\mathrm{TM}}$. Hierfür wird der Gesamtzeitraum von 1998-2017 betrachtet, der dann nochmal sind 5-Jahresabschnitte aufgeteilt wurde. Abb. 4 zeigt die concept map für die 20 Jahre mit den häufigsten Konzepten in den Artikeln. Die Cluster oder thematischen Regionen sind jeweils nach den in ihnen am häufigsten vorkommenden Konzepten benannt. Dies sind „sozial“ ( $\mathrm{h}=278$ Treffer; $100 \%)$, gefolgt von „empirisch“ ( $\mathrm{h}=225 ; 81 \%)$, „Schule“ $(\mathrm{h}=220 ; 79 \%)$, ,Bildung“ $(\mathrm{h}=206 ; 74 \%)$, „Lernen“ $(\mathrm{h}=102 ; 37 \%)$ und „Unterricht“ $(\mathrm{h}=88 ; 32 \%)^{7}$.

Betrachten wir den Gesamtzeitraum so steht also auch hier das Konzept „sozial“ besonders im Mittelpunkt: der soziale Hintergrund von Kindern im Hinblick auf ihre Entwicklung (siehe Konzeptpfad Bildung-sozial-Merkmale-Kinder-Eltern-Entwicklung) und Kompetenzentwicklung (Entwicklung-Kompetenzen-Schüler). Dominanter Bildungskontext in den veröffentlichten Studien in der ZfE ist die Schule: „Schule“ oder „Schüler“ sind in allen Zeiträumen deutlich vertreten, während zum Beispiel Arbeiten aus dem Bereich der Weiterbildung oder des lebenslangen Lernens nicht sichtbar sind. Die empirische Bildungsforschung erscheint besonders prominent an

Abb. 8 Concept Map erstellt mit dem Textmining Tool Leximancer $^{\mathrm{TM}}$ auf Grundlage der Abstracts der ZfE in den Jahren 2013-2017 $(N=306)$

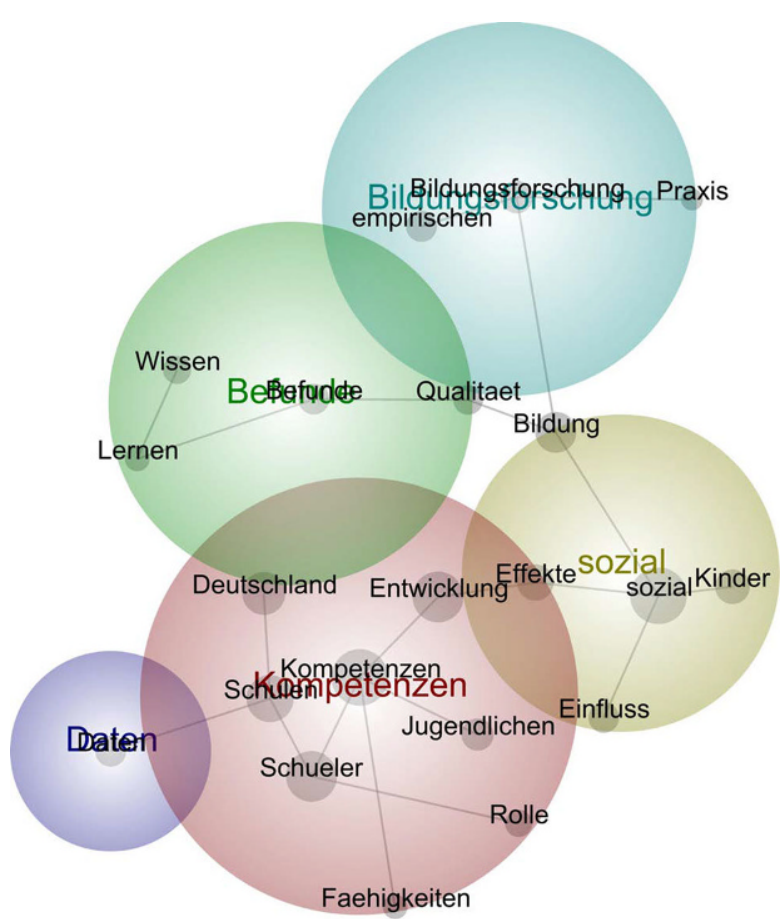

\footnotetext{
7 Die Größe der Kreise um die thematischen Regionen herum ergibt sich in der Darstellung von Leximancer $^{\mathrm{TM}}$ aus der Anzahl der darin vorkommenden Konzepte. Die Häufigkeit des am häufigsten vorkommenden Konzeptes (hier ,sozial“ (278 mal vorkommend)) wird auf 100\% gesetzt. Die Prozentangaben der folgenden Konzepte beziehen sich auf diesen Wert.
} 
der Schnittstelle zu pädagogischen Handlungsfeldern (Bildungsforschung-pädagogischen-Praxis) und im Kontext von Lernen und Unterrichten (empirisch-WissenLernen-Unterricht).

In den folgenden concept maps finden sich immer wiederkehrende Konzepte, die so allgemein sind, dass sie für eine Interpretation wenig aussagekräftig sind (z.B. ,pädagogisch“, „Lernen“, „Gesellschaft“ oder „Bildung“). Vielmehr wird in der anschließenden Darstellung auf neu erscheinende oder auch auffällig nicht mehr vorkommende Konzepte eingegangen. Dabei veranschaulichen wir die vier Zeiträume durch Literaturbelege, die jedoch eher einen illustrierenden Charakter haben und keinen Anspruch darauf erheben, die publizierten Studien in ihrer Vollständigkeit abzubilden.

\subsubsection{Gründungsjahre einer erziehungswissenschaftlichen Zeitschrift (1998-2002)}

Im Zeitraum von 1998-2002 (siehe Abb. 5) sind die thematischen Regionen benannt nach den Konzepten ,pädagogischen“ ( $h=45 ; 100 \%)$, ,sozial“ ( $h=39 ; 87 \%)$, „Gesellschaft“ $(\mathrm{h}=26 ; 58 \%)$, „Erziehungswissenschaft“ $(\mathrm{h}=33 ; 73 \%)$ und ,Alter“ $(\mathrm{h}=14 ; 31 \%)$.

Mit der Verbreitung des Internets und der sogenannten neuen Informations- und Kommunikationstechnologien Ende der 1990er-Jahre beschäftigen sich in der thematischen Region des „Pädagogischen“, viele Beiträge mit Medien, insbesondere mit den ,neuen Medien“. Schorb (1998) führt das Stichwort „Medienpädagogik“ in der ersten Ausgabe der ZfE ein. Von Hentig (1998) diskutiert die Entwicklung einer Medien-Generation und versucht diese zu charakterisieren. Er geht der Frage nach, wie das Leben in der Medien-Generation aussieht und welche Folgen daraus resultieren (Medien-Entwicklung-Jugendlichen). Andere Beiträge nehmen die Medienkompetenz von Schülerinnen und Schülern als Informationsverwertungskompetenz (Imai 1998) in den Blick und sprechen das „Gefälle zwischen dem medienorientierten Kinderalltag und dem traditionellen Schulalltag“ (S. 61) an. Breiter (2001) betrachtet digitale Medien im Schulsystem und deren organisatorische Einbettung in Deutschland, den USA und Großbritannien.

Das Konzept „,sozial“ liegt an der Schnittstelle zwischen „Medien“, „Bildung“ und „Schulen“. Beiträge adressieren u.a. Aspekte der Familienbildung und Jugendhilfe (Schiersmann und Thiel 1999), der Freizeitnutzung sozialer Räume durch Jugendliche (Merkens 2001) oder der Begriffsbestimmung von Inklusion und Exklusion in der sozialen Arbeit (Merten 2001). Die kleine thematische Region „Alter“ ist über die Konzepte „Menschen“ und „Bildung“ mit „sozial“ verbunden. Stellvertretend beschäftigt sich der Aufsatz von Kruse (2001) mit dem Beitrag der Erwachsenenbildung zur Kompetenz im Alter.

\subsubsection{Reaktionen auf PISA (2003-2007)}

In den folgenden fünf Jahren (2003-2007) sind die häufigsten Konzepte gruppiert um ,sozial“" $(\mathrm{h}=55 ; 100 \%)$ und ,Schule“ $(\mathrm{h}=47 ; 85 \%)$, gefolgt von „Bildung“ $(\mathrm{h}=40 ; 73 \%)$, „PISA“ $(\mathrm{h}=15 ; 27 \%)$ und ,Überzeugungen“ $(\mathrm{h}=14 ; 25 \%$; siehe Abb. 6). 
Nach dem Schock der PISA-Studie 2000 ist das Programm zur internationalen Schülerbewertung nun auch deutlich in das Interesse der Beiträge in der ZfE gerückt, insbesondere der Zusammenhang zwischen sozialen Strukturmerkmalen familiärer Lebensverhältnisse und der Bildungsbeteiligung und dem Kompetenzerwerb (PISAHerkunft-sozial-Kinder-Eltern, Baumert et al. 2003). Watermann und Maaz (2006) stellen fest, dass durch PISA die sozialen Ungleichheiten der Bildungsbeteiligung in das Zentrum der bildungssoziologischen Diskussion gerückt sind. Koch (2004) stellt im Vergleich zum Humboldt'schen Konzept Allgemeiner Menschenbildung infrage, ob das in der PISA-Studie aus dem angelsächsischen entlehnte literacy-Konzept eine neue Art von Allgemeinbildung und Grundbildung oder nur ein allgemeines Qualifikationskonzept darstellt (Deutschland-Konzept-Grundbildung-Bildung). Vor dem Hintergrund der PISA-Ergebnisse richtet Kansanen (2004) den Blick auf das finnische Curriculum der Lehrerbildung, wobei auch fachliche Überzeugungen und Haltungen der Lehrkräfte als Schlüssel für guten Unterricht einbezogen werden. Die Überzeugungen von Lehrkräften sind Gegenstand weiterer Artikel, z. B. im Hinblick auf das handlungsleitende Lehr-Lernverständnis (Leuchter et al. 2006) oder das Sprachenlernen (Trautmann 2005; siehe Konzeptpfad: Lehrkräfte-SchülerUnterricht-Überzeugungen).

\subsubsection{Hinwendung zu einer evidenzbasierten Praxis (2008-2012)}

In der concept map von 2008-2012 sind die häufigsten Konzepte ,sozial“ ( $\mathrm{h}=75$; $100 \%)$, „Schüler“ $(\mathrm{h}=41 ; 55 \%)$, „Pädagogik“ $(\mathrm{h}=39 ; 52 \%)$ und „Deutschland“ $(\mathrm{h}=32 ; 43 \%)$. Im Zusammenhang mit ,empirisch“ tritt „Praxis“ $(\mathrm{h}=19 ; 25 \%)$ als thematische Region auf (siehe Abb. 7).

In den Beiträgen von 2008-2012 wird eine Phase eingeläutet, in der empirische Befunde im Sinne einer evidence-based policy (vgl. Slavin 2002), die pädagogische Praxis und auch bildungspolitische Entscheidungen in Deutschland informieren sollen (siehe Pädagogik-Praxis-empirisch/Deutschland). So betonen Pietsch und Tosana (2008) im Kontext von Schulinspektionen, dass es wichtig ist, ,,durch den Einsatz angemessener empirischer Verfahren eine fundierte Qualitätssicherung zu etablieren, die es gestattet, Probleme bei der Messung von Unterrichtsqualität sichtbar zu machen“ (S. 430). Pietsch (2010) legt ein mehrstufiges Modell zur Evaluation von Unterrichtsqualität vor (Schule-Unterricht-Daten). Die Förderung einer empirisch ausgerichteten Bildungsforschung fokussiert jedoch nicht nur den Schulkontext, sondern ist auf Bildungsverläufe und Kompetenzentwicklungsprozesse entlang der ganzen Lebensspanne angelegt. Besonders hervorzuheben ist hier die Einrichtung des Nationalen Bildungspanels (NEPS) durch das Bundesministerium für Bildung und Forschung, in dem seit 2009 längsschnittliche Daten erhoben werden (Blossfeld et al. 2011).

\subsubsection{Etablierung der empirischen Bildungsforschung (2013-2017)}

Die Orientierung zur empirischen Bildungsforschung setzt sich in den folgenden fünf Jahren (2013-2017) deutlich fort. Das häufigste Konzept ist weiterhin ,sozi-

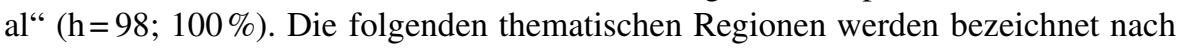


den Konzepten „Kompetenzen“ $(\mathrm{h}=83 ; 85 \%)$, „Daten“ $(\mathrm{h}=47 ; 48 \%)$, „Befunde“ $(\mathrm{h}=43 ; 44 \%)$ und „Bildungsforschung“ ( $\mathrm{h}=39 ; 40 \%$; siehe Abb. 8).

Der Einfluss sozialer Merkmale und deren Effekte auf die Entwicklung von Kompetenzen und Fähigkeiten auf Kinder und Jugendliche ist ein dominantes Thema in den Veröffentlichungen (Einfluss-sozial-Effekte-Entwicklung-Kompetenzen). Hillmert (2014) präsentiert empirische Befunde in Bezug auf soziale Ungleichheit, Bildungszugang und Bildungserwerb im Lebenslauf. Fuchs-Rechlin und Bergmann (2014) setzen bei der frühkindlichen Bildung, Betreuung und Erziehung an und gehen der Frage nach, inwieweit der sozioökonomische Status der Familien und ein Migrationshintergrund unter dreijähriger Kinder ihre Teilhabe an Kindertagesbetreuungsangeboten beeinflusst. Dieser verliert mit zunehmenden Alter der Kinder an Wirkung. Klapprodt et al. (2014) nehmen den Schulformwechsel in der Sekundarstufe in den Blick und stellen fest, dass sich die Wahrscheinlichkeit eines Schulwechsels auf eine höhere Schulform unabhängig vom Migrationshintergrund und den Schulnoten mit den elterlichen Bildungsaspirationen erhöht.

In Verbindung zu „Bildung“ tritt wieder der Konzeptpfad empirischen-Bildungsforschung-Praxis auf. Es wird angestrebt, mit den Befunden der empirischen Forschung, die Qualität von Bildungsprozessen zu verbessern (Lernen-Befunde-Qualität-Bildung). Ein Schwerpunkt wird auf die Kompetenzentwicklung angehender Lehrkräfte gelegt, da diese maßgeblich die Gestaltung und Qualität von Unterricht beeinflussen. So legen z. B. Kuhn et al. (2014) für Lehrkräfte im kaufmännischverwaltenden Bereich ein domänenspezifisches Kompetenzmodell und ein Messinstrument vor, mit dem sie den Zusammenhang zwischen Fachwissen und fachdidaktischem Wissen untersuchen. Wurster et al. (2017) können jedoch keinen direkten signifikanten Zusammenhang zwischen datenbasierter Unterrichtsentwicklung und den Schülerleistungen feststellen (Daten-Schulen). Eine skeptische Haltung zum Nutzen der PISA-Studien als Entscheidungsgrundlage für eine evidenzbasierte Bildungspolitik nimmt Klemm (2016) ein, da Befunde zum Teil unterschiedlich oder sogar widersprüchlich interpretiert und somit unterschiedliche Handlungsempfehlungen abgeleitet werden und einzelne Ergebnisse wenig belastbar sind. Von einem „schwierigen Verhältnis“ (S. 23) zwischen Bildungsforschung und Bildungstheorie nach PISA spricht auch Messner (2016). Er kommt jedoch zu der Erkenntnis, dass in der neu empirisch vermessenden Bildungslandschaft nicht die zu erwartende Polarität zwischen Bildungstheorie und Bildungsforschung besteht: „Es findet sich vielmehr eine Pluralität von teils konkurrierenden, sich teils ergänzenden Wissenschaftsformen alten und neuen Typs vor“" (S. 23).

\section{Diskussion und Fazit}

Im Folgenden werden wir zunächst die Forschungsliteratur noch einmal zusammenfassen und vor diesem Hintergrund unsere Ergebnisse diskutieren. Die erziehungswissenschaftliche Forschungslandschaft hat sich im deutschsprachigen Raum in den letzten zwanzig Jahren, insbesondere durch den enormen Aufschwung der empirischen Bildungsforschung stark weiterentwickelt. Diese Entwicklung wurde von verschiedenen Autor/innen anhand der Denomination von Professuren (Krüger 
et al. 2008), dem Anteil der in SOFIS gelisteten Forschungsprojekte (Sondergeld und Botte 2013) und der Teilhabe an Drittmittelprojekten (Köller 2014) quantitativ nachgezeichnet. Es liegen auch bereits Arbeiten vor, die sich mit der inhaltlichen Schwerpunktsetzung erziehungswissenschaftlicher Fachpublikationen beschäftigen (Dees und Botte 2013b; Kauder 2014), aber eine entsprechende inhaltliche Verschiebung der Schwerpunktsetzung kaum nachweisen können. Angesichts des zwanzigjährigen Jubiläums der $Z f E$ haben wir im vorliegenden Beitrag die ZfE-Beiträge der letzten zwanzig Jahre mit Methoden der quantitativen Inhalts- und Textanalyse ausgewertet. Diese Analysen ermöglichen keine Wirkungsforschung, können jedoch Hinweise darauf liefern, inwiefern sich Tendenzen, die in der erziehungswissenschaftlichen Forschungslandschaft zu beobachten sind, auch in einer renommierten erziehungswissenschaftlichen Fachzeitschrift, wie der ZfE widerspiegeln.

Die Ergebnisse der Inhaltsanalyse mit dem Textmining Tool Leximancer ${ }^{\mathrm{TM}}$ korrespondieren mit denen der quantitativen Textanalyse. Dabei zeigt sich in den concept maps und in der quantitativen Textanalyse, dass die Begriffe bzw. Konzepte ${ }^{8}$ „Pädagogik“ und „Erziehungswissenschaft“" in der Etappe zwischen 1998 und 2002 prominenter vertreten sind als in den drei folgenden Etappen und die Bigramme ,allgemeine Erziehungswissenschaft“" und ,,allgemeine Pädagogik“ - wie die tf-idfWerte zeigen - besonders charakteristisch für die Gründungsjahre der Zeitschrift sind. Dabei setzen sich die Beiträge im Gründungszeitraum intensiv mit den Aufgaben und der Leistungsfähigkeit der allgemeinen Pädagogik und Erziehungswissenschaft auseinander und diskutieren das Verhältnis zu anderen Subdisziplinen und zur empirischen Bildungsforschung (z.B. Ehrenspeck 1998; Tippelt 1998; Vogel 1998). Hingegen zählen die Bigramme ,empirische Forschung“ bzw. „empirische Bildungsforschung" kontinuierlich bereits in der ersten Etappe und auch in den drei weiteren Etappen zu den von den Autorinnen und Autoren der ZfE am häufigsten verwendeten Bigrammen, wenngleich sich über die zwanzig Jahre hinweg ein leichter Aufwärtstrend in der Verwendung des Begriffes ,Bildungsforschung“ zeigt (Abb. 3). Entsprechend ist in den concept maps im ersten Zeitraum zwischen 1998 und 2002 das Konzept „Erziehungswissenschaft“ eine eigene thematische Region, während im vierten Zeitraum zwischen 2013 und 2017 das Konzept „Bildungsforschung“ eine eigene thematische Region bildet. Dies deutet darauf hin, dass die Autorinnen und Autoren der $Z f E$ ihre Beiträge zunehmend in der empirischen Bildungsforschung verorten. Dieses Ergebnis mag die Stärkung der empirischen Bildungsforschung (Köller 2014) widerspiegeln, die in den letzten fünfundzwanzig Jahren verstärkt Fördermittel erhielt und zunehmend den Forschungsschwerpunkt der universitären Stellen bildete (Köller 2014; Krüger et al. 2008; Schmidt-Hertha und Tippelt 2014).

Darüber hinaus wird deutlich, dass das Konzept „,sozial“ über die gesamte Zeitspanne sehr prominent in der ZfE vertreten ist. So ist es in allen vier Zeitspannen eines der häufigsten Konzepte in den concept maps. In der ersten Etappe zwischen 1998 und 2002 kann das Konzept „sozial“ an der Schnittstelle zwischen „Medien“, „Bildung“ und „Schulen“ verortet werden und tritt u.a. im Zusammenhang mit der

\footnotetext{
8 Im Zusammenhang mit der quantitativen Textanalyse sprechen wir hier von „Begriffen“, während die häufig in den Texten vorkommenden und miteinander in Beziehung stehenden Wörter im Rahmen der Inhaltsanalyse als „Konzepte“ bezeichnet werden.
} 
Inklusion in der sozialen Arbeit (Merten 2001) und der Nutzung sozialer Räume durch Jugendliche (Merkens 2001) auf. In den darauffolgenden Etappen wird - wie die Betrachtung der häufigsten Bigramme zeigt - hingegen vorrangig die ,soziale Herkunft" bzw. ,soziale Ungleichheit" in den Beiträgen der ZfE untersucht. Dies mag durch die Ergebnisse der PISA-Studie mitbedingt sein, in denen die Rolle der sozialen Herkunft für den Bildungserfolg deutscher Schülerinnen und Schüler deutlich wurde (z. B. Watermann und Maaz 2006).

Weiterhin zeigen unsere Ergebnisse, dass die ZfE-Artikel im gesamten Zeitraum vorrangig auf den schulischen Kontext fokussieren. So sind in den concept maps die Begriffe „Schule“ oder „Schüler“ deutlich vertreten, während beispielsweise Aspekte des lebenslangen Lernens seltener angeführt werden. Auch die quantitativen Analysen der Wortstämme zeigen, dass Schülerinnen und Schüler häufiger im Fokus der ZfE-Artikel stehen als Lehrkräfte, die aber wiederum deutlich häufiger Gegenstand der Untersuchung sind als Schulleiterinnen und Schulleiter. Dieser Fokus auf die unmittelbar am Unterrichtsgeschehen Beteiligten entspricht zumindest dem engeren Verständnis von Bildungsforschung, wie es vom deutschen Bildungsrat 1974 geprägt wurde. Darin wird konstatiert, dass es Bildungsforschung ,im engeren Sinne [...] als Unterrichtsforschung schon immer gegeben" habe (Deutscher Bildungsrat 1974, S. 23). Die Analyse der Wortstämme deutet zudem darauf hin, dass Studierende in den $Z f E$-Beiträgen seltener thematisiert werden als Lehrkräfte und als Schülerinnen und Schüler, wobei sich ein leichter Aufwärtstrend in der Fokussierung auf Studierende über die letzten beiden Zeiträume zeigt.

\subsection{Limitationen und Forschungsimplikationen}

Die vorliegende Studie weist einige Limitationen auf. Aus unseren Analysen lassen sich gewisse Hinweise auf die Entwicklung der inhaltlichen Ausrichtung der Publikationen in der $Z f E$ ableiten. Die Befunde können aber nicht ohne weiteres auf die gesamte deutschsprachige erziehungswissenschaftliche Publikationslandschaft verallgemeinert werden. Dees und Botte (2013a) haben darauf hingewiesen, dass die Generalisierbarkeit von bibliometrischen Analysen stets von den der Analyse zugrundeliegenden Zeitschriften oder Fachdatenbanken abhängig ist. Die Analyse von Artikeln einer Fachzeitschrift mit bestimmten Qualitätskriterien, wie dem Peer-Review - so wie im vorliegenden Beitrag - stellt zwar eine gewisse Qualität der eingeschlossenen Artikel sicher. Andererseits kann sie die Vielfalt der Publikationstypen und der Publikationsmedien der erziehungswissenschaftlichen Publikationstätigkeit nicht hinreichend abbilden. Eine zusätzliche Einschränkung bei der Interpretation unserer Ergebnisse ergibt sich aus den unterschiedlichen Publikationspraktiken, die in verschiedenen Subdisziplinen der Erziehungswissenschaft üblich sind (Dees und Botte 2013b). Dees und Botte (2013b) konnten beispielsweise zeigen, dass in der Erwachsenenbildung anteilig seltener in Fachzeitschriften publiziert wird als in der Schulforschung. Die Ergebnisse zur Publikationstätigkeit, die wir im vorliegenden Beitrag darstellen, können zudem auch als Folge von politischen Entscheidungen interpretiert werden (Abs und Kuper 2020). Die Interpretation, dass sich die Autor/ innen der ZfE-Beiträge mit ihrer Forschung zunehmend in der Bildungsforschung verorten, wäre daher auch anders lesbar. So wäre bspw. auch denkbar, dass Beiträge 
mit einem bildungswissenschaftlichen Fokus von den Gutachter/innen im Peer-Review häufiger als qualitativ hinreichend, aber auch als passend für eine Publikation in der ZfE angesehen werden. Entsprechend weisen Abs und Kuper (2020) auf folgenden Punkt hin, der bei der Rezeption unserer Ergebnisse berücksichtigt werden muss: „Die auf empirische Daten gestützte Beschreibung bietet einen Bezugspunkt, von dem ausgehend Bewertungen, Entwicklungsperspektiven und vielleicht sogar Handlungsoptionen zu gewinnen sind, die allerdings bei der Größe, Heterogenität und Komplexität des Faches aus unterschiedlichen Interessenpositionen vorgenommen werden“" (S. 18).

Darüber hinaus bieten quantitative Textanalysen, wie wir sie im vorliegenden Beitrag vorgenommen haben erfolgsversprechende Möglichkeiten, um große Textmengen personal- und/oder zeitökonomisch zu analysieren. Sie weisen aber auch einige Unschärfen auf. Denn anhand der Nennung von Wortstämmen lässt sich nicht fehlerfrei feststellen, inwiefern sich ein Beitrag tatsächlich einer spezifischen Subdisziplin zuordnen lässt. Um zu bestimmen, welche Anteile einzelne Subdisziplinen einnehmen, könnten zukünftige Studien auch manuelle Kodierungen vornehmen und diese mit den Ergebnissen aus quantitativen Textanalysen abgleichen. Zudem ist der Vergleich mit Ergebnissen, die anhand der Analysen von Schlagwörtern in Fachdatenbanken gewonnen werden (z. B. Sondergeld und Botte 2013) gewinnbringend. $\mathrm{Zu}$ beachten ist auch, dass eine Unterteilung in Zeitabschnitte - wie wir sie im vorliegenden Beitrag vorgenommen haben - nur arbiträr sein kann. Die Analyse der Häufigkeit von Wortstämmen, die auf einzelnen Jahrgängen beruht, soll die Aussagen, die auf der Grundlage der Fünfjahresabschnitte getroffen werden, ergänzen. Sicher wäre jedoch auch eine andere Unterteilung der Zeiträume denkbar. Grundsätzlich lässt sich festhalten, dass es einer zusätzlichen validierenden kulturhistorischen Analyse des Soziotops „Wissenschaft“ bedürfte, die der vorliegende Beitrag nicht leisten kann, um bruchlos auf disziplinäre Ausdifferenzierungs- und Formierungsprozesse folgern zu können.

Schließlich ist anhand quantitativer Methoden nur schwer feststellbar, inwiefern sich bestimmte Analyseansätze über die Zeit verändert haben. Bspw. wäre ein Vergleich der relativen Häufigkeit des Wortstamms ,"längsschnitt“ und „querschnitt“ vermutlich irreführend, da in den Abstracts insbesondere die spezifischen Stärken der Beiträge hervorgehoben werden. Durch spezifische Weiterentwicklungen von FIS Bildung oder anderen erziehungswissenschaftlichen Fachdatenbanken - bspw. einer Verschlagwortung der angewandten Forschungsmethoden - wäre die Analyse von Trends in der methodischen Ausrichtung von Forschungsarbeiten zuverlässiger und ökonomischer leistbar (Dees und Botte 2013a).

\subsection{Fazit}

Insgesamt lässt sich festhalten, dass unsere Analysen auf einige Kontinuitäten in den Publikationen der ZfE über den untersuchten Zeitraum von zwanzig Jahren hinweg hindeuten. So ist methodisch durchweg ein Fokus auf empirische Forschung und eine gehäuft auftretende inhaltliche Ausrichtung auf soziale Aspekte von Bildung sichtbar. Dieses Ergebnis ist konsistent mit den Befunden von Kauder (2014), die nahelegen, dass die inhaltliche Schwerpunktsetzung der erziehungswissenschaftlichen 
Dissertationen, die an deutschen, österreichischen und schweizerischen Hochschulen entstanden im Zeitraum 1990-1999 ähnlich war, wie im Zeitraum 2000-2009. Die von uns gefundenen Hinweise auf eine gewisse Kontinuität in der inhaltlichen Ausrichtung der $Z f E$ deckt sich zudem mit den Befunden von Dees und Botte (2013b), die zeigten, dass sich im Zeitraum 1998-2002 ein ähnlicher Anteil der Publikationen der Literaturdatenbank FIS-Bildung den Subdisziplinen Fachdidaktik, Erwachsenenbildung, Sonderpädagogik, oder Bildungsforschung und -theorie zuordnen ließen wie zwischen 2003 und 2007. Andererseits deuten unsere Analysen auch darauf hin, dass sich die Terminologie bzw. die inhaltliche Verortung der ZfE-Autorinnen und -Autoren insofern geändert hat als dass im Gründungszeitraum vermehrt die Bigramme „Allgemeine Erziehungswissenschaft“ und „Allgemeine Pädagogik“ erwähnt wurden, die in der Folgezeit seltener auftraten. Der Begriff „Bildungsforschung" findet sich zwar im gesamten Untersuchungszeitraum, wird jedoch mit den Jahren tendenziell häufiger verwendet. Diese veränderte Ausrichtung entspricht der Wahrnehmung von Gräsel (2011), die konstatiert hatte, dass sich die zunehmende Förderung und institutionelle Verankerung der empirischen Bildungsforschung in der Universitätslandschaft auch in der erziehungswissenschaftlichen Publikationstätigkeit wiederfindet. Der Befund ist auch stimmig mit bibliometrischen Analysen der im Forschungsinformationssystem SOFIS verzeichneten bildungswissenschaftlichen Forschungsprojekte, die zwischen 1995 und 2006 anteilig zugenommen haben (Sondergeld und Botte 2013). Schließlich hat sich die Publikationskultur insofern geändert, dass Manuskripte in zunehmenden Maße von mehreren Autorinnen und Autoren gestaltet werden und der Anteil an Autorinnen über die Jahre gestiegen ist. Dies ist konsistent mit internationalen Entwicklungen (West et al. 2013; ZawackiRichter und von Prümmer 2010). 


\section{Anhang}

Abb. 9 Mittlere Anzahl der Autorinnen und Autoren pro Beitrag der ZfE in den Jahren 1998-2017 (dargestellt sind gleitende Mittelwerte über ein Zeitfenster von fünf Jahren)

Abb. 10 Prozentualer Anteil der ZfE-Beiträge in den Jahren 1998-2017, an denen ein bis drei oder mehr Autorinnen und $\mathrm{Au}$ toren beteiligt waren (dargestellt sind gleitende Mittelwerte über ein Zeitfenster von fünf Jahren)
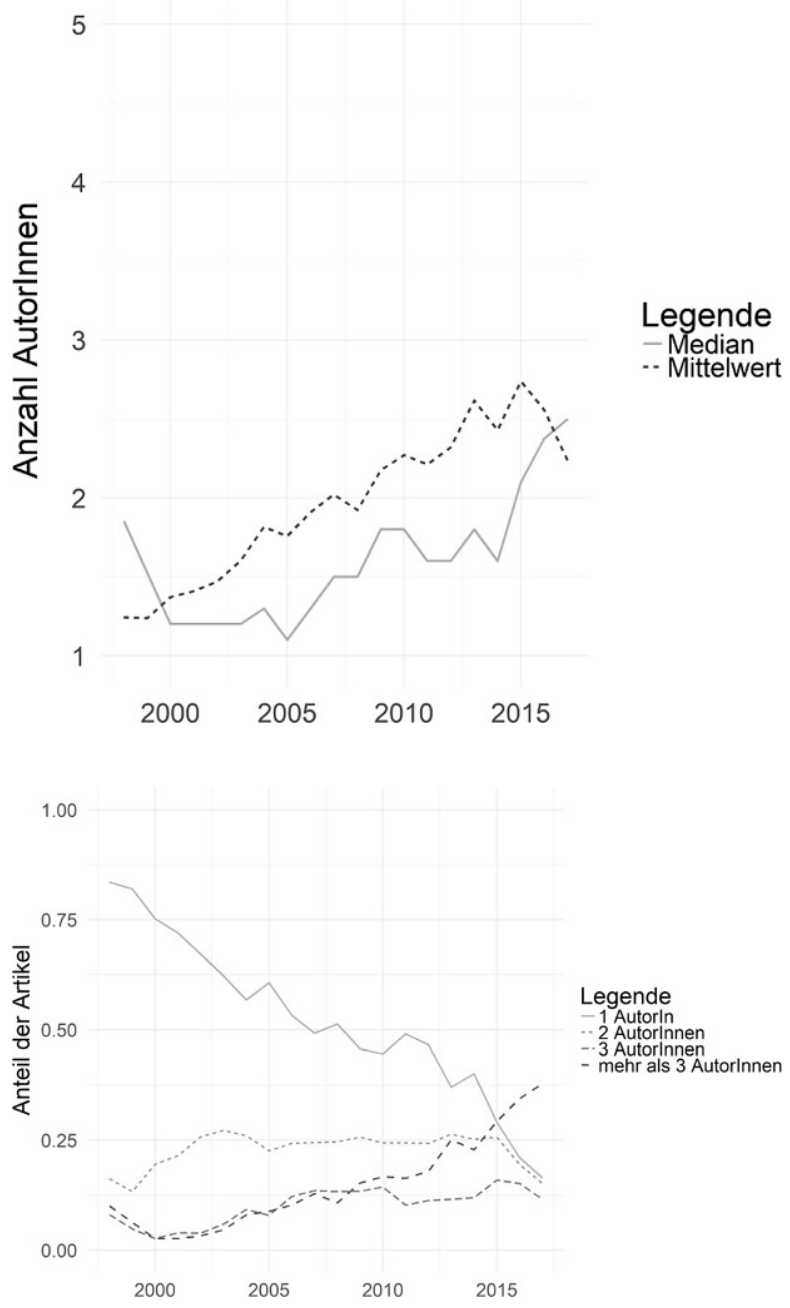
Abb. 11 Prozentualer Anteil der ZfE-Beiträge in den Jahren 1998-2017 mit weiblicher Beteiligung und weiblicher Erstautorin (dargestellt sind gleitende Mittelwerte über ein Zeitfenster von fünf Jahren)

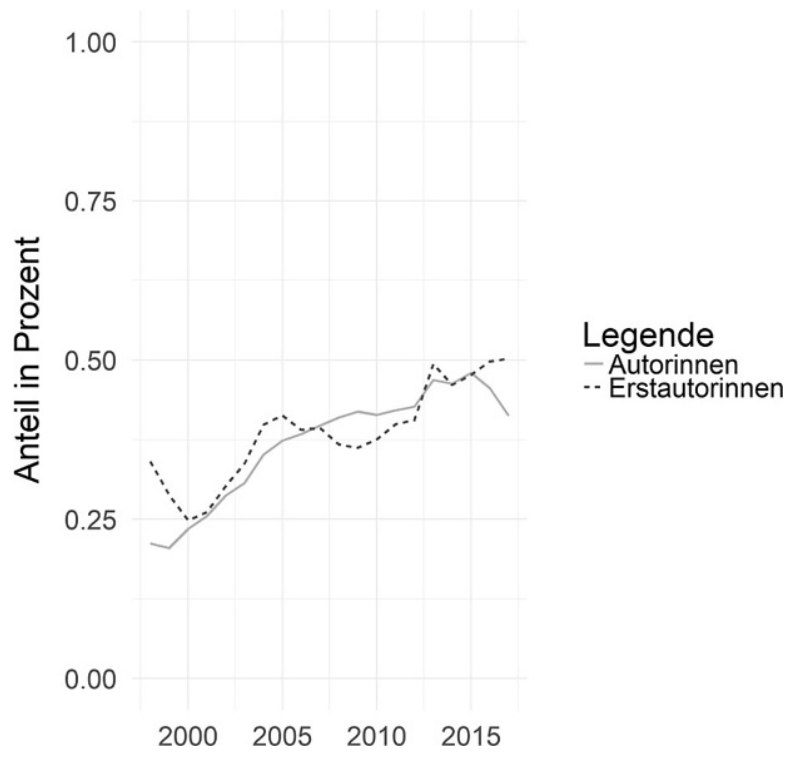

Tab. 1 Institutionelle Zugehörigeit der ZfE-Autorinnen und Autoren aufgeschlüsselt nach Ländern im Zeitraum 1998-2017

\begin{tabular}{|c|c|c|}
\hline Land & Artikelanzahl & Prozent \\
\hline Deutschland & 709 & 86,4 \\
\hline Schweiz & 36 & 4,4 \\
\hline USA & 18 & 2,2 \\
\hline Großbritanien & 17 & 2,1 \\
\hline Niederlande & 15 & 1,8 \\
\hline Frankreich & 12 & 1,5 \\
\hline Japan & 5 & 0,6 \\
\hline Österreich & 5 & 0,6 \\
\hline Belgien & 4 & 0,5 \\
\hline Korea & 4 & 0,5 \\
\hline Luxemburg & 4 & 0,5 \\
\hline Finnland & 3 & 0,4 \\
\hline Norwegen & 3 & 0,4 \\
\hline Schweden & 3 & 0,4 \\
\hline Kanada & 2 & 0,2 \\
\hline Spanien & 2 & 0,2 \\
\hline Australien & 1 & 0,1 \\
\hline Israel & 1 & 0,1 \\
\hline Mexiko & 1 & 0,1 \\
\hline Tschechische Republik & 1 & 0,1 \\
\hline
\end{tabular}

Prozentzahlen entsprechen dem Anteil an Beiträgen, an denen ein Land beteiligt war Prozentzahlen addieren sich zu mehr als $100 \%$, weil an einigen Beiträgen mehrere Institutionen beteiligt waren 
Tab. 2 Institutionen mit der höchsten Anzahl an ZfE-Beiträgen im Zeitraum 1998-2017

\begin{tabular}{lll}
\hline Institution & Artikelanzahl & Prozent \\
\hline Freie Universität Berlin & 69 & 8,4 \\
Humboldt-Universität zu Berlin & 46 & 5,6 \\
Universität Hamburg & 38 & 4,6 \\
Deutsches Institut für Internationale Pädagogische Forschung & 36 & 4,4 \\
Universität Bamberg & 30 & 3,7 \\
Technische Universität München & 26 & 3,2 \\
Leibniz-Institut für die Pädagogik der Naturwissenschaften und & 24 & 2,9 \\
Mathematik & & 2,7 \\
Martin-Luther-Universität Halle-Wittenberg & 22 & 2,2 \\
Max-Planck-Institut Für Bildungsforschung & 18 & 2,1 \\
Eberhard Karls Universität Tübingen & 17 & 2,1 \\
Technische Universität Dortmund & 17 & 1,9 \\
Ludwig-Maximilians-Universität München & 16 & 1,9 \\
Max-Plnack-Institut für Bildungsforschung & 16 & 1,9 \\
Universität Tübingen & 16 & 1,8 \\
Leibniz-Institut für die Pädagogik der Naturwissenschafeten und & 15 & \\
Mathematik & & \\
\hline
\end{tabular}

Prozentzahlen entsprechen dem Anteil an Beiträgen, an denen eine Institution beteiligt war

Prozentzahlen addieren sich zu mehr als $100 \%$, weil an einigen Beiträgen mehrere Institutionen beteiligt waren

Tab. 3 Autorinnen und Autoren mit der höchsten Anzahl an ZfE-Beiträgen im Zeitraum 1998-2017

\begin{tabular}{ll}
\hline AutorIn & Artikelanzahl \\
\hline Lüdtke, Oliver & 16 \\
Trautwein, Ulrich & 15 \\
Maaz, Kai & 14 \\
Kunter, Mareike & 11 \\
Nagy, Gabriel & 11 \\
Baumert, Jürgen & 10 \\
Köller, Olaf & 10 \\
Neumann, Marko & 9 \\
Prenzel, Manfred & 9 \\
Klieme, Eckhard & 8 \\
Stanat, Petra & 8 \\
Blömeke, Sigrid & 7 \\
Blossfeld, Hans-Peter & 7 \\
Ehmke, Timo & 7 \\
Helsper, Werner & 7 \\
König, Johannes & 7 \\
Kramer, Jochen & 7 \\
Seidel, Tina & 7 \\
Thiel, Felicitas & 7 \\
Watermann, Rainer & 7 \\
Zirfas, Jörg & 7 \\
\hline
\end{tabular}


Funding Open Access funding enabled and organized by Projekt DEAL.

Open Access Dieser Artikel wird unter der Creative Commons Namensnennung 4.0 International Lizenz veröffentlicht, welche die Nutzung, Vervielfältigung, Bearbeitung, Verbreitung und Wiedergabe in jeglichem Medium und Format erlaubt, sofern Sie den/die ursprünglichen Autor(en) und die Quelle ordnungsgemäß nennen, einen Link zur Creative Commons Lizenz beifügen und angeben, ob Änderungen vorgenommen wurden.

Die in diesem Artikel enthaltenen Bilder und sonstiges Drittmaterial unterliegen ebenfalls der genannten Creative Commons Lizenz, sofern sich aus der Abbildungslegende nichts anderes ergibt. Sofern das betreffende Material nicht unter der genannten Creative Commons Lizenz steht und die betreffende Handlung nicht nach gesetzlichen Vorschriften erlaubt ist, ist für die oben aufgeführten Weiterverwendungen des Materials die Einwilligung des jeweiligen Rechteinhabers einzuholen.

Weitere Details zur Lizenz entnehmen Sie bitte der Lizenzinformation auf http://creativecommons.org/ licenses/by/4.0/deed.de.

\section{Literatur}

Abs, H. J., \& Kuper, H. (2020). Datenreport Erziehungswissenschaft - zwanzig Jahre indikatorenbasiertes Monitoring der Disziplin. In H.-J. Abs, H. Kuper \& R. Martini (Hrsg.), Datenreport Erziehungswissenschaft 2020. Erstellt im Auftrag der Deutschen Gesellschaft für Erziehungswissenschaft (DGfE) (S. 9-20). Opladen: Barbara Budrich.

Baumert, J., Watermann, R., \& Schümer, G. (2003). Disparitäten der Bildungsbeteiligung und des Kompetenzerwerbs: Ein institutionelles und individuelles Mediationsmodell. Zeitschrift für Erziehungswissenschaft, 6(1), 46-71. https://doi.org/10.1007/s11618-003-0004-7.

Bedenlier, S., Kondakci, Y., \& Zawacki-Richter, O. (2018). Two decades of research into the internationalization of higher education: Major themes in the Journal of Studies in International Education (1997-2016). Journal of Studies in International Education, 22(2), 108-135. https://doi.org/10.1177/ 1028315317710093.

Blossfeld, H.-P., von Maurice, J., \& Schneider, T. (2011). The National Educational Panel Study: need, main features, and research potential. Zeitschrift Für Erziehungswissenschaft, 14(1), 5-17. https:// doi.org/10.1007/s11618-011-0178-3.

Botte, A. (2014). The relevance of the EERQI framework in the light of future perspectives: enhancing the visibility and detection of European research publications. In I. Gogolin, F. Åström \& A. Hansen (Hrsg.), Assessing quality in European educational research (S. 184-196). Wiesbaden: Springer VS.

Botte, A. (2015). Publikationskultur in Erziehungswissenschaft und Fachdidaktik. Eine empirische Annäherung. In K. Sommer, J. Lorke \& C. Mattiesson (Hrsg.), Publizieren in Zeitschriften für Forschung und Unterrichtspraxis (S. 136-153). Bad Heilbrunn: Klinkhardt.

Botte, A., \& Rittberger, M. (2016). Bildung und Bildungsforschung in Massenmedien. Ein Forschungsszenario. Pädagogische Rundschau, 70(2), 159-174.

Breiter, A. (2001). Digitale Medien im Schulsystem: Organisatorische Einbettung in Deutschland, den USA und Großbritannien. Zeitschrift für Erziehungswissenschaft, 4(4), 625-639. https://doi.org/10. 1007/s11618-001-0061-8.

Cretchley, J., Rooney, D., \& Gallois, C. (2010). Mapping a 40-year history with leximancer: Themes and concepts in the journal of cross-cultural psychology. Journal of Cross-cultural Psychology, 41(3), $318-328$.

Dees, W. (2013). „Publication power approach“. Ein neuer Ansatz zur Bewertung von Publikationsorten der Erziehungswissenschaft? In H.-P. Ohly (Hrsg.), Wissen, Wissenschaft, Organisation (S. 392-401). Würzburg: Ergon.

Dees, W., \& Botte, A. (2013a). Publikationspraxis in der Bildungsforschung 1998-2007. Frankfurt a. M.: Deutsches Institut für Internationale Pädagogische Forschung.

Dees, W., \& Botte, A. (2013b). Veröffentlichungen in der FIS Bildung Literaturdatenbank. In H. Weishaupt \& M. Rittberger (Hrsg.), Bildungsforschung in Deutschland-eine Situationsanalyse. Berlin: BMBF.

Deutscher Bildungsrat (1974). Aspekte für die Planung der Bildungsforschung (Empfehlungen der Bildungskommission, Bd. 1). Stuttgart: Klett. 
Ehrenspeck, Y. (1998). Teildisziplinen ohne Allgemeine Erziehungswissenschaft? Folgen unterlassener Reflexion, Begriffskritik und Grundlagenforschung beim Theorieimport „Alltag“. Zeitschrift für Erziehungswissenschaft, 1(2), 181-201.

Fatke, R., \& Oelkers, J. (2014). Das Selbstverständnis der Erziehungswissenschaft: Geschichte und Gegenwart. Geschichte und Gegenwart. Einleitung zum Beiheft. In R. Fatke \& J. Oelkers (Hrsg.), Das Selbstverständnis der Erziehungswissenschaft: Geschichte und Gegenwart (Zeitschrift für Pädagogik: Beiheft Bd. 60, S. 7-13). Weinheim: Beltz.

Fuchs-Rechlin, K., \& Bergmann, C. (2014). Der Abbau von Bildungsbenachteiligung durch Kindertagesbetreuung für unter 3-Jährige - zwischen Wunsch und Wirklichkeit. Zeitschrift für Erziehungswissenschaft, 17(2), 95-118. https://doi.org/10.1007/s11618-013-0464-3.

Gallmann, M. E. (2018). Unschärfe in der Erziehungswissenschaft: Bilanzierung einer Wissensform. Opladen: Barbara Budrich.

Gogolin, I., Kuper, H., Krüger, H., \& Baumert, J. (2013). Editorial. In I. Gogolin, H. Kuper, H.-H. Krüger \& J. Baumert (Hrsg.), Stichwort: Zeitschrift für Erziehungswissenschaft (S. 7-11). Wiesbaden: Springer.

Gräsel, C. (2011). Was ist Empirische Bildungsforschung? In H. Reinders, H. Ditton, C. Gräsel \& B. Gniewosz (Hrsg.), Strukturen und Methoden (Empirische Bildungsforschung, Bd. 1, S. 13-28). Wiesbaden: Verlag für Sozialwissenschaften.

von Hentig, H. (1998). Jugend im Medienzeitalter. Zeitschrift Für Erziehungswissenschaft, 1(1), $23-43$.

Hillmert, S. (2014). Bildung, Ausbildung und soziale Ungleichheiten im Lebenslauf. Zeitschrift für Erziehungswissenschaft, 17(2), 73-94. https://doi.org/10.1007/s11618-013-0465-2.

Horn, K.-P. (2003). Erziehungswissenschaft in Deutschland im 20. Jahrhundert. Zur Entwicklung der sozialen und fachlichen Struktur der Disziplin von der Erstinstitutionalisierung bis zur Expansion. Bad Heilbrunn: Klinkhardt.

Imai, Y. (1998). Neue Medien im Spiegel der pädagogischen Diskussion in Japan. Zeitschrift Für Erziehungswissenschaft, 1(1), 61-72.

Kansanen, P. (2004). The role of general education in teacher education. Zeitschrift Für Erziehungswissenschaft, 7(2), 207-218. https://doi.org/10.1007/s11618-004-0022-0.

Kauder, P. (2014). Themenkonjunkturen im Spiegel erziehungswissenschaftlicher Dissertationen und Habilitationen 1945-2009. In R. Fatke \& J. Oelkers (Hrsg.), Das Selbstverständnis der Erziehungswissenschaft: Geschichte und Gegenwart (Zeitschrift für Pädagogik: Beiheft Bd. 60, S. 184-207). Weinheim: Beltz.

Keiner, E. (1999). Erziehungswissenschaft 1947-1990. Eine empirische und vergleichende Untersuchung zur kommunikativen Praxis einer Diszipliny. Weinheim: Deutscher Studien Verlag.

Klapproth, F., Schaltz, P., \& Glock, S. (2014). Elterliche Bildungsaspiration und Migrationshintergrund als Prädiktoren für Schulformwechsel in der Sekundarstufe I: Ergebnisse einer Längsschnittstudie. Zeitschrift für Erziehungswissenschaft, 17(2), 323-343.

Klemm, K. (2016). Die PISA-Studien: Ihre Präsentation und Interpretation im Lichte der Evidenzbasierung. Zeitschrift für Erziehungswissenschaft, 19(1), 163-177. https://doi.org/10.1007/s11618-0160710-6.

Koch, L. (2004). Allgemeinbildung und Grundbildung, Identität oder Alternative? Zeitschrift für Erziehungswissenschaft, 7(2), 183-191. https://doi.org/10.1007/s11618-004-0020-2.

Köller, O. (2014). Entwicklung und Erträge der jüngeren empirischen Bildungsforschung. In R. Fatke \& J. Oelkers (Hrsg.), Das Selbstverständnis der Erziehungswissenschaft: Geschichte und Gegenwart (Zeitschrift für Pädagogik: Beiheft Bd. 60, S. 102-122). Weinheim: Beltz.

Krüger, H.-H., Schnorr, O., \& Weishaupt, H. (2008). Personal. In K.-J. Tillmann, T. Rauschenbach, R. Tippelt \& H. Weishaupt (Hrsg.), Datenreport Erziehungswissenschaft 2008. Opladen: Barbara Budrich.

Kruse, A. (2001). Der Beitrag der Erwachsenenbildung zur Kompetenz im Alter. Zeitschrift für Erziehungswissenschaft, 4(4), 555-575. https://doi.org/10.1007/s11618-001-0057-4.

Kuhn, C., Happ, R., Zlatkin-Troitschanskaia, O., Beck, K., Förster, M., \& Preuße, D. (2014). Kompetenzentwicklung angehender Lehrkräfte im kaufmännisch-verwaltenden Bereich - Erfassung und Zusammenhänge von Fachwissen und fachdidaktischem Wissen. Zeitschrift für Erziehungswissenschaft, 17(1), 149-167. https://doi.org/10.1007/s11618-013-0456-3.

Lenzen, D. (1998). Eine neue erziehungswissenschaftliche Zeitschrift! Zeitschrift für Erziehungswissenschaft, 1(1), 3-5.

Leuchter, M., Pauli, C., Reusser, K., \& Lipowsky, F. (2006). Unterrichtsbezogene Überzeugungen und handlungsleitende Kognitionen von Lehrpersonen. Zeitschrift für Erziehungswissenschaft, 9(4), 562-579. https://doi.org/10.1007/s11618-006-0168-z. 
Lin, J., \& Lee, S. T. (2012). Mapping 12 years of communication scholarship: themes and concepts in the journal of communication. In H.-H. Chen \& G. Chowdhury (Hrsg.), The outreach of digital libraries: A globalized resource network (S. 359-360). Berlin: Springer.

Merkens, H. (2001). Die Nutzung sozialer Räume durch Jugendliche in ihrer Freizeit. Zeitschrift für Erziehungswissenschaft, 4(3), 437-455. https://doi.org/10.1007/s11618-001-0046-7.

Merten, R. (2001). Inklusion/Exklusion und Soziale Arbeit: Überlegungen zur aktuellen Theoriedebatte zwischen Bestimmung und Destruktion. Zeitschrift für Erziehungswissenschaft, 4(2), 173-190. https://doi.org/10.1007/s11618-001-0022-2.

Messner, R. (2016). Bildungsforschung und Bildungstheorie nach PISA - ein schwieriges Verhältnis. Zeitschrift für Erziehungswissenschaft, 19(1), 23-44. https://doi.org/10.1007/s11618-016-0706-2.

Pietsch, M. (2010). Evaluation von Unterrichtsstandards. Zeitschrift für Erziehungswissenschaft, 13(1), 121-148. https://doi.org/10.1007/s11618-010-0113-z.

Pietsch, M., \& Tosana, S. (2008). Beurteilereffekte bei der Messung von Unterrichtsqualität: Das Multifacetten-Rasch-Modell und die Generalisierbarkeitstheorie als Methoden der Qualitätssicherung in der externen Evaluation von Schulen. Zeitschrift für Erziehungswissenschaft, 11(3), 430-452. https://doi. org/10.1007/s11618-008-0021-7.

Röbken, H., Zawacki-Richter, O., \& Zierer, K. (2013). Zwischen Vielfalt und Einheit. Eine Expertenbefragung zu deutschsprachigen Zeitschriften der Erziehungswissenschaft. Erziehungswissenschaft, 24(46), 73-83.

Rost, F. (2013). Stichwort: Zeitschrift für Erziehungswissenschaft (ZfE). In I. Gogolin, H. Kuper, H.H. Krüger \& J. Baumert (Hrsg.), Stichwort: Zeitschrift für Erziehungswissenschaft (S. 13-30). Wiesbaden: Springer.

Roth, H. (1963). Die realistische Wendung in der pädagogischen Forschung. Die Deutsche Schule, 55(3), 109-119.

Schiersmann, C., \& Thiel, H.-U. (1999). Innovationen in der Familienbildung. Ergebnisse einer bundesweiten Institutionenanalyse. Zeitschrift Für Erziehungswissenschaft, 2(1), 99-113.

Schmidt-Hertha, B., \& Tippelt, R. (2014). Erziehungswissenschaft und das Verhältnis zu ihren Bezugsdisziplinen vor dem Hintergrund der Stellen- und Besetzungspraxis (1995 bis 2012). In R. Fatke \& J. Oelkers (Hrsg.), Das Selbstverständnis der Erziehungswissenschaft: Geschichte und Gegenwart (Zeitschrift für Pädagogik: Beiheft Bd. 60, S. 172-183). Weinheim: Beltz.

Schmidt-Hertha, B., \& Müller, M. (2020). Forschung und Publikationskulturen. In H.-J. Abs, H. Kuper \& R. Martini (Hrsg.), Datenreport Erziehungswissenschaft 2020. Erstellt im Auftrag der Deutschen Gesellschaft für Erziehungswissenschaft (DGfE) (S. 147-170). Opladen: Barbara Budrich.

Schorb, B. (1998). Stichwort: Medienpädagogik. Zeitschrift Für Erziehungswissenschaft, 1(1), 7-22.

Schulze, T. (2002). Allgemeine Erziehungswissenschaft und erziehungswissenschaftliche Biographieforschung. In L. Wigger (Hrsg.), Forschungsfelder der Allgemeinen Erziehungswissenschaft (S. 129-146). Opladen: Leske \& Budrich.

Silge, J., \& Robinson, D. (2016). tidytext: text mining and analysis using tidy data principles in R. The Journal of Open Source Software. https://doi.org/10.21105/joss.00037.

Slavin, R.E. (2002). Evidence-based education policies: transforming educational practice and research. Educational Researcher, 31(7), 15-21. https://doi.org/10.3102/0013189X031007015.

Smith, A.E., \& Humphreys, M. S. (2006). Evaluation of unsupervised semantic mapping of natural language with Leximancer concept mapping. Behavior Research Methods, 38(2), 262-279. https://doi.org/ 10.3758/BF03192778.

Sondergeld, U., \& Botte, A. (2013). Monitoring Bildungsforschung: Mehrdimensionale szientometrische Untersuchung eines interdisziplinären Forschungsfeldes. In H.-C. Hobohm (Hrsg.), Informationswissenschaft zwischen virtueller Infrastruktur und materiellen Lebenswelten. Tagungsband des 13. Internationalen Symposiums für Informationswissenschaft (ISI 2013) (S. 180-191). Potsdam, 19.-22. März 2013. Glückstadt: Werner Hülsbusch.

Tippelt, R. (1998). Zum Verhältnis von allgemeiner Pädagogik und empirischer Bildungsforschung. Zeitschrift für Erziehungswissenschaft, 1(2), 239-260.

Trautmann, M. (2005). Überzeugungen vom Englischlernen. Zeitschrift für Erziehungswissenschaft, 8(1), 38-52. https://doi.org/10.1007/s11618-005-0122-5.

Vogel, P. (1998). Stichwort: Allgemeine Pädagogik. Zeitschrift für Erziehungswissenschaft, 1(2), 157-180.

Watermann, R., \& Maaz, K. (2006). Effekte der Öffnung von Wegen zur Hochschulreife auf die Studienintention am Ende der gymnasialen Oberstufe. Zeitschrift für Erziehungswissenschaft, 9(2), 219-239. https://doi.org/10.1007/s11618-006-0019-y.

Weishaupt, H., \& Rittberger, M. (2013). Bildungsforschung in Deutschland - eine Situationsanalyse. Berlin: BMBF. 
West, J.D., Jacquet, J., King, M. M., Correll, S. J., \& Bergstrom, C. T. (2013). The role of gender in scholarly authorship. PloS one, 8(7), e66212. https://doi.org/10.1371/journal.pone.0066212.

Wurster, S., Richter, D., \& Lenski, A. E. (2017). Datenbasierte Unterrichtsentwicklung und ihr Zusammenhang zur Schülerleistung. Zeitschrift für Erziehungswissenschaft, 20(4), 628-650. https://doi.org/10. 1007/s11618-017-0759-x.

Zawacki-Richter, O., \& Naidu, S. (2016). Mapping research trends from 35 years of publications in Distance Education. Distance Education, 37(3), 245-269.

Zawacki-Richter, O., \& von Prümmer, C. (2010). Gender and collaboration patterns in distance education research. Open Learning, 25(2), 95-114.

Züll, C., \& Mohler, P. P. (1992). Was ist TEXTPACK? In C. Züll \& P. P. Mohler (Hrsg.), Textanalyse: Anwendungen der computerunterstützten Inhaltsanalyse (S. 225-227). Opladen: Westdeutscher Verlag.

Züll, C., \& Landmann, J. (2002). Computerunterstïtzte Inhaltsanalyse: Literaturbericht zu neueren Anwendungen. Mannheim: Zentrum für Umfragen, Methoden und Analysen -ZUMA. https:// nbnresolving.org/urn:nbn:de:0168-ssoar-52606-9. Zugegriffen: 18. Juni 2020. 